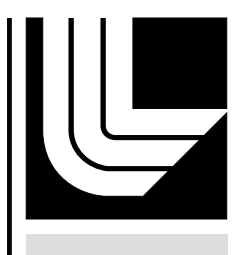

LAW RENCE LIVERMORE N A TION A L LABORATORY

\title{
UCRL-TR-206882
}

\section{Ultrasonic Calibration Wire Test Phantom}

S. K. Lehman, K. A. Fisher, M. Werve, D. H. Chambers

September 29, 2004 
This document was prepared as an account of work sponsored by an agency of the United States Government. Neither the United States Government nor the University of California nor any of their employees, makes any warranty, express or implied, or assumes any legal liability or responsibility for the accuracy, completeness, or usefulness of any information, apparatus, product, or process disclosed, or represents that its use would not infringe privately owned rights. Reference herein to any specific commercial product, process, or service by trade name, trademark, manufacturer, or otherwise, does not necessarily constitute or imply its endorsement, recommendation, or favoring by the United States Government or the University of California. The views and opinions of authors expressed herein do not necessarily state or reflect those of the United States Government or the University of California, and shall not be used for advertising or product endorsement purposes.

This work was performed under the auspices of the U.S. Department of Energy by University of California, Lawrence Livermore National Laboratory under Contract W-7405-Eng-48. 


\title{
Ultrasonic Calibration Wire Test Phantom
}

\author{
Sean K. Lehman, Karl A. Fisher, Mick Werve, David H. Chambers \\ February 15, 2005
}

\begin{abstract}
We designed and built a phantom consisting of vertical wires maintained under tension to be used as an ultrasonic test, calibration, and reconstruction object for the Lawrence Livermore National Laboratory annular array scanner. We provide a description of the phantom, present example data sets, preliminary reconstructions, example metadata, and MATLAB codes to read the data.
\end{abstract}

UCRL-TR-206882 


\section{Side View}

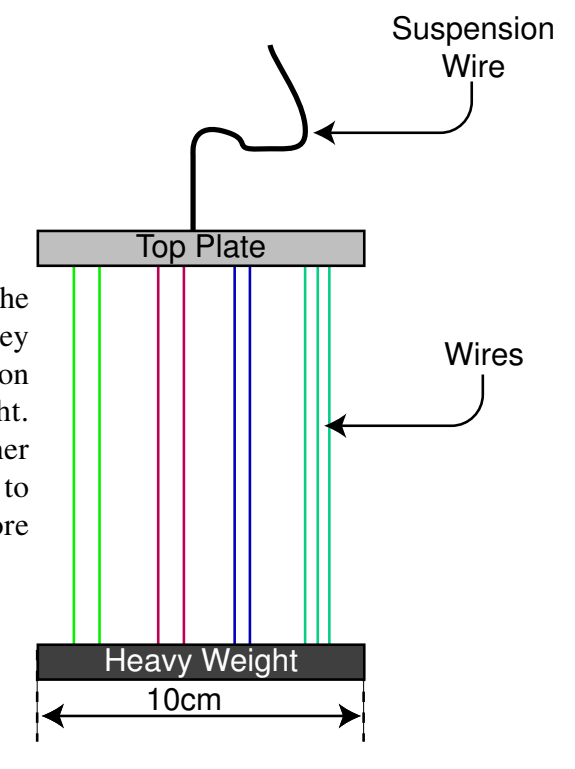

Removable outer cylinder. Slips over the suspended wire phantom. Data will be collected without and with the sheath.

The sheath will be fastened to the weight in a water tight manner. The phantom may then be filled with a contrast agent.

\section{Top View}
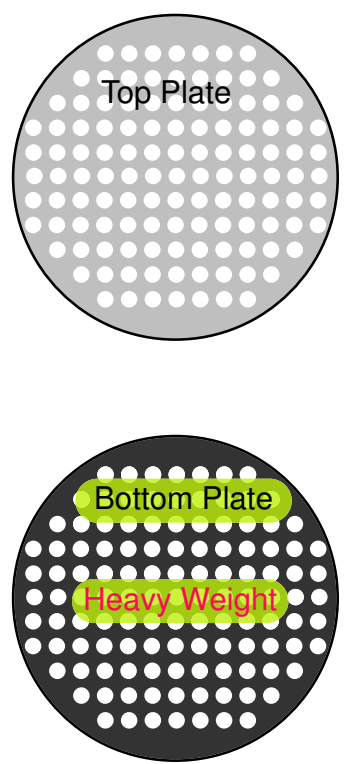

Wires of varying material properties will be threaded through the plate holes and arranged in various patterns.

Figure 1: Preliminary wire phantom conceptual design. 


\section{Introduction}

We designed and built a reconfigurable wire phantom for the ultrasonic Lawrence Livermore National Laboratory annular array scanner. We collected well characterized data under controlled conditions for use as "canonical data sets" in testing and evaluating new inversion algorithms.

The phantom consists of vertical wires maintained under tension. The planar, $(x, y)$, wire distribution is governed by holes drilled through upper and lower wire guide plates in a selected geometric pattern. The wires are threaded through the holes and secured to mounting brackets. Given that the phantom object formed by the wire distribution does not vary much in the vertical ( $z$-direction), we assume the measured data form a 2.5-dimensional object. The initial conceptual phantom design is presented in Figure 1

These plates are removable permitting differing designs to be used. We currently only have one design: a logarithmic spiral with equation,

$$
\mathbf{r}\left(\theta_{n}\right)=a\left(\cos \left(\theta_{n}\right), \sin \left(\theta_{n}\right)\right) e^{b \theta_{n}}
$$

where

$$
\begin{aligned}
a & \equiv \lambda_{0} & & \text { is the initial radius and, } \\
\lambda_{0} & \equiv v_{0} / f_{0} & & \text { is the insonifying wavelength, } \\
v_{0} & \equiv 1500 \text { meters/second } & & \text { is the assumed background water velocity, } \\
f_{0} & \equiv 1 \mathrm{MHz} & & \text { is the approximate insonifying frequency, } \\
b & \equiv 10 \pi / 180 & & \text { is the spiral growth rate, } \\
\theta_{n} & \equiv\{n \Delta \theta\}_{n=0}^{N-1} & & \text { are the angular locations of the wires, } \\
\Delta \theta & \equiv 30 \pi / 180 & & \text { is the angular increment, } \\
N & \equiv 31 & & \text { is the number of wires. }
\end{aligned}
$$

This current configuration is shown in Figure 2(a). We have allowed for an optional acrylic hollow cylinder around the phantom as shown in Figure 2(b). Figure 2(c) shows one of the plates. The $(x, y)$ locations of the wires in the logarithmic spiral are listed in Table 1. Figure 3 shows a graphic of the phantom in relation to the transducer locations.

Figures 4 and 5 show the phantom under construction while being installed in the scanner. The top block is affixed to a rod fastened to the central bore of the scanner drive; the bottom block is fastened to the bottom of the tank. Figure 6 (a) shows the phantom with 31 taut steel wires. Figure 6 (b) shows the optional acrylic cylinder in place.

The design of the phantom allows for wires of different materials to be used. We have run scans with the following combinations:

- 31 steel wires;

- 30 steel, 1 nylon;

- 25 steel, 6 nylon;

- 25 steel, 6 nylon, with the hollow cylinder;

- 2 steel, 2 nylon resolution pair;

- 2 steel, 2 nylon resolution pair, with the hollow cylinder;

- Water background (no wires); 


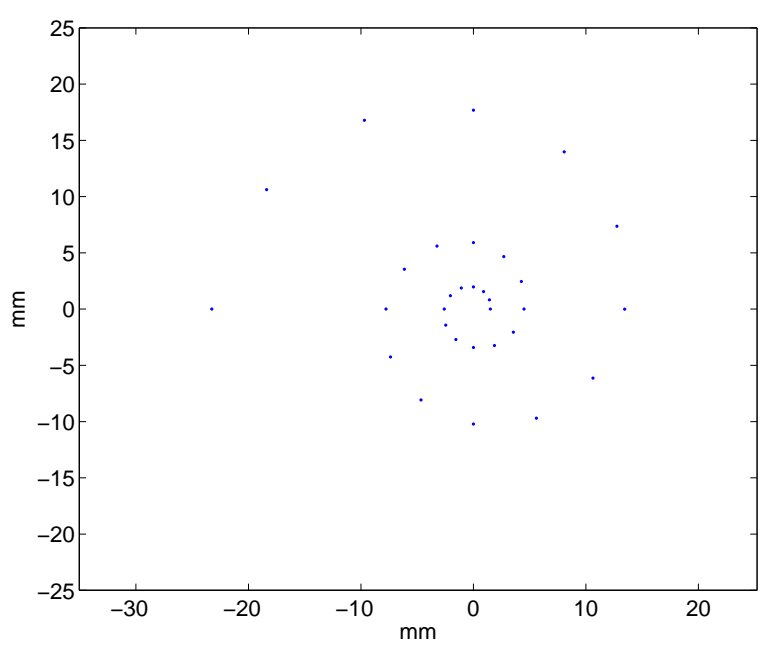

(a)

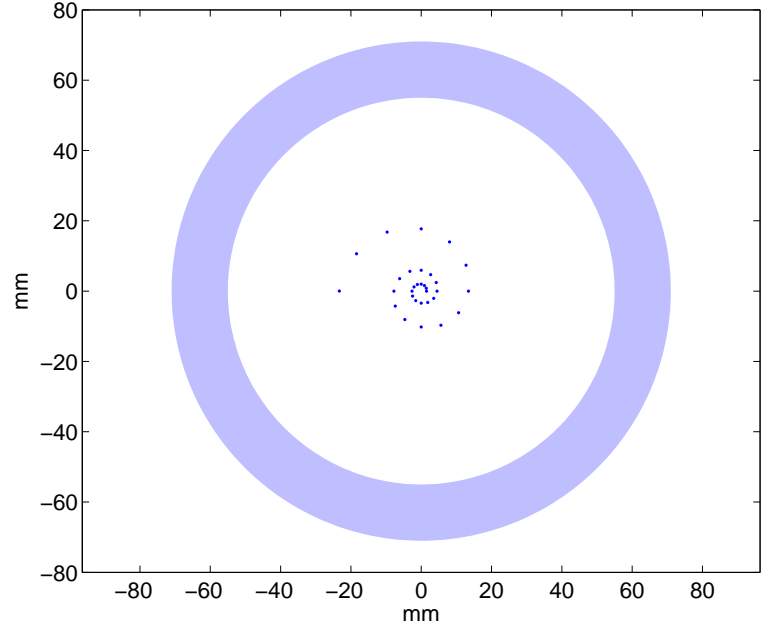

(b)

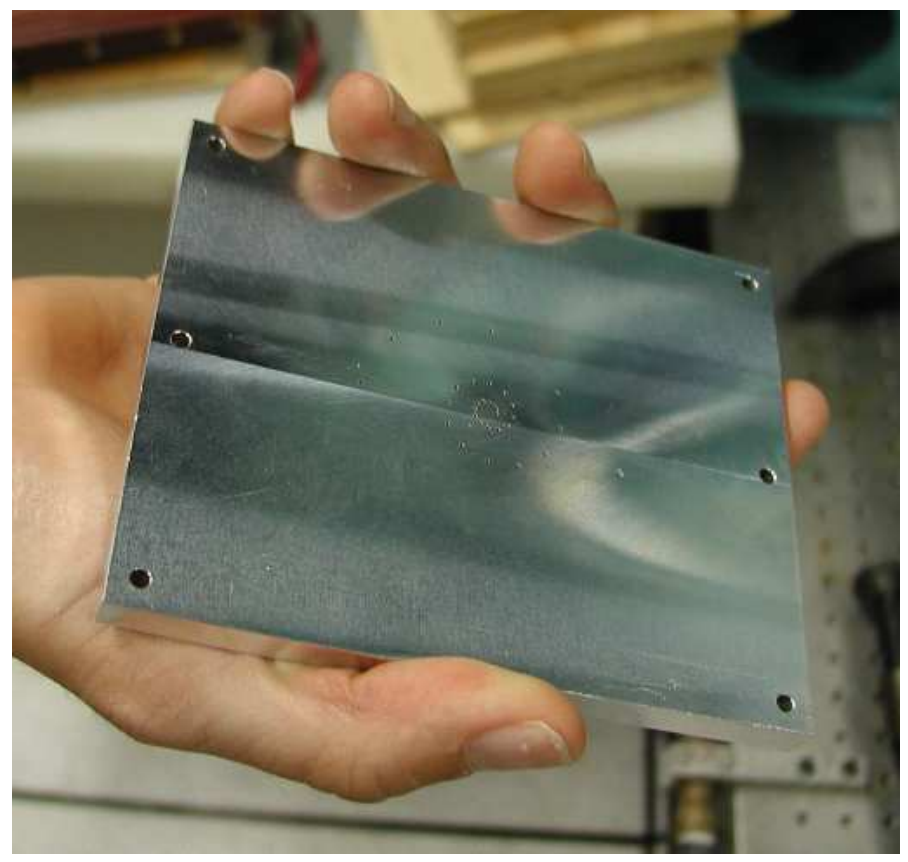

(c)

Figure 2: Basic spiral phantom design. (a) Without hollow cylinder. (b) With cylinder. (c) Photograph of wiring plate with holes. 


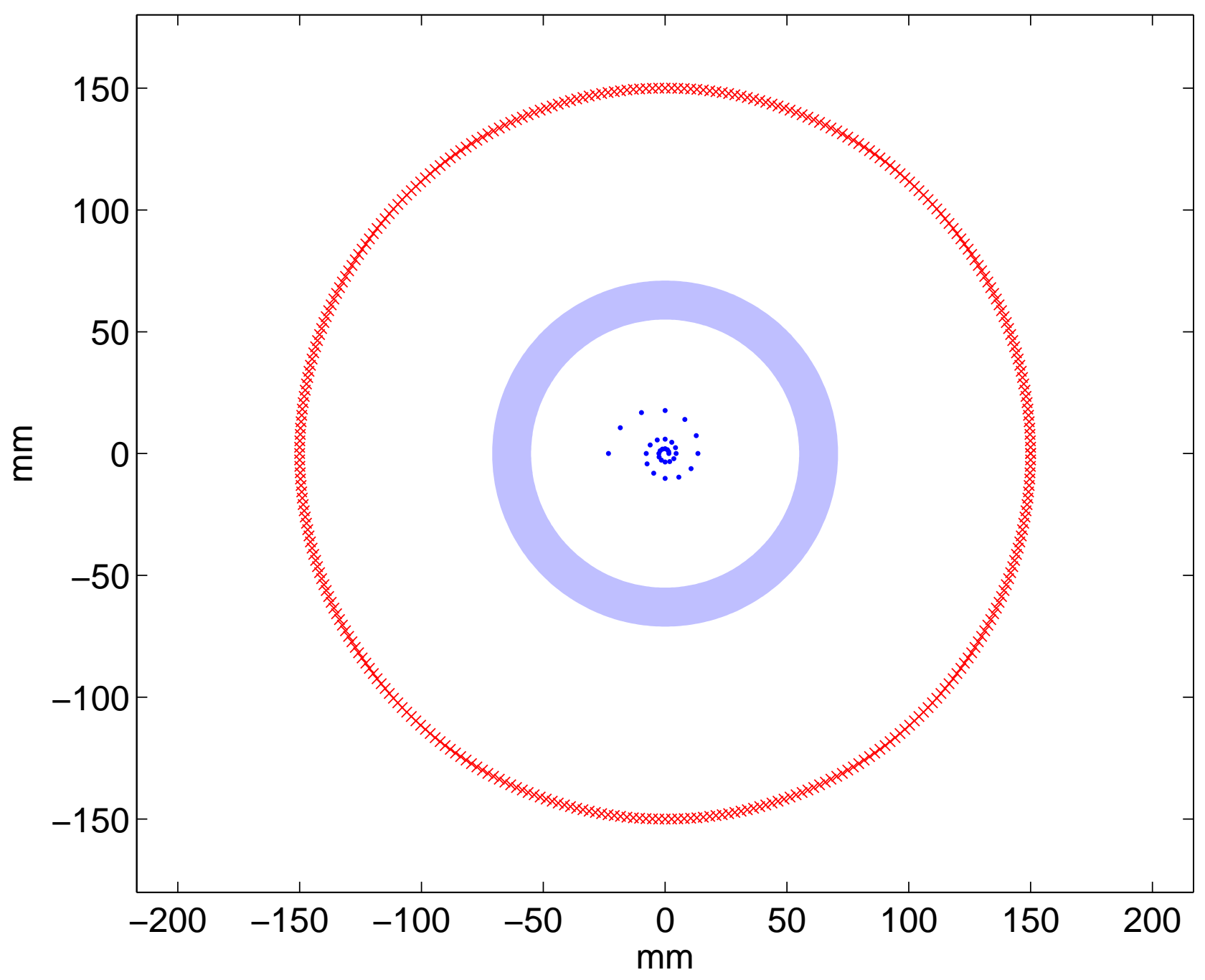

Figure 3: Graphic showing phantom in relation to transducer array location. 


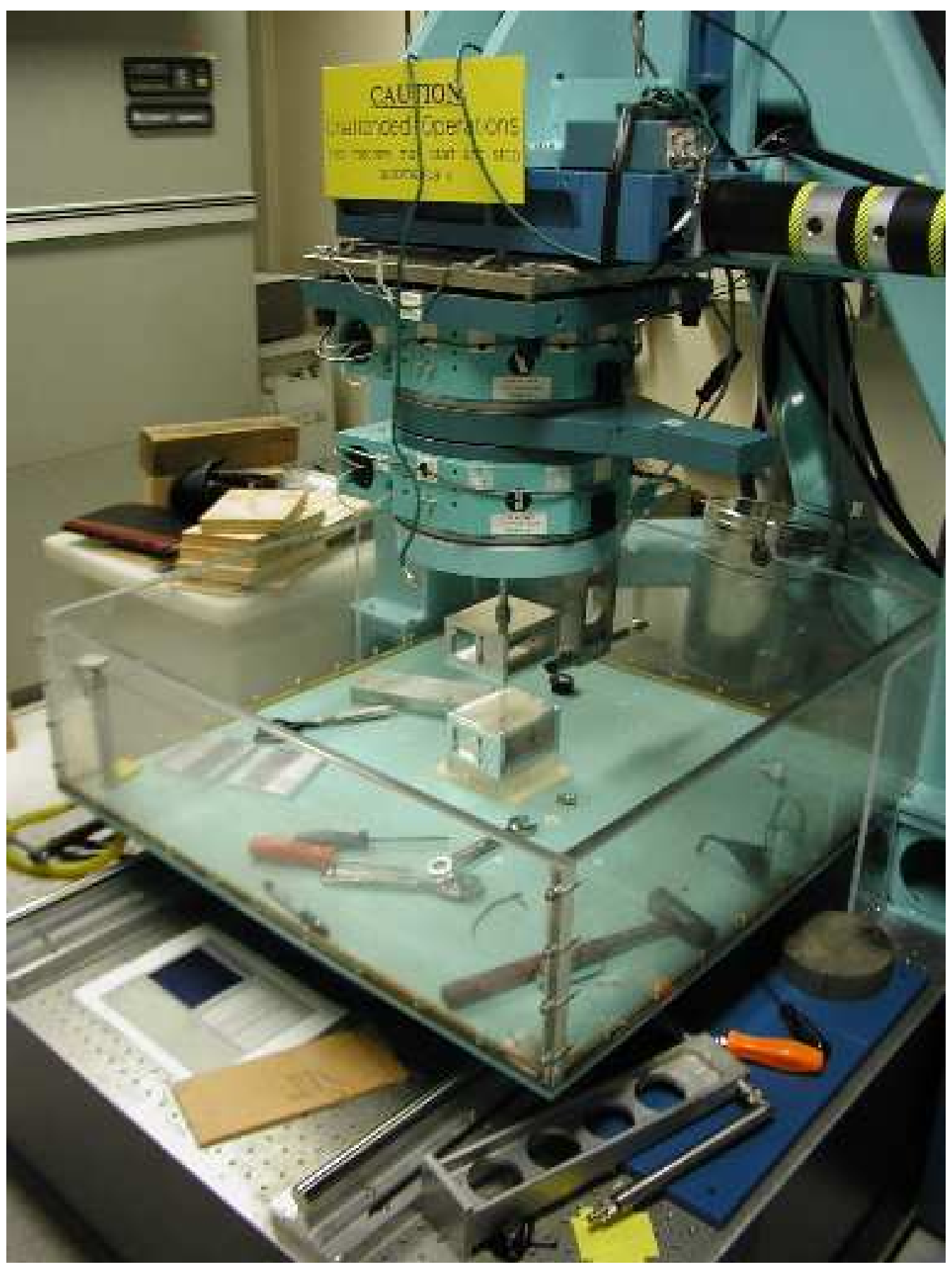

Figure 4: Phantom under construction during installation in ultrasonic LLNL annular array scanner. 


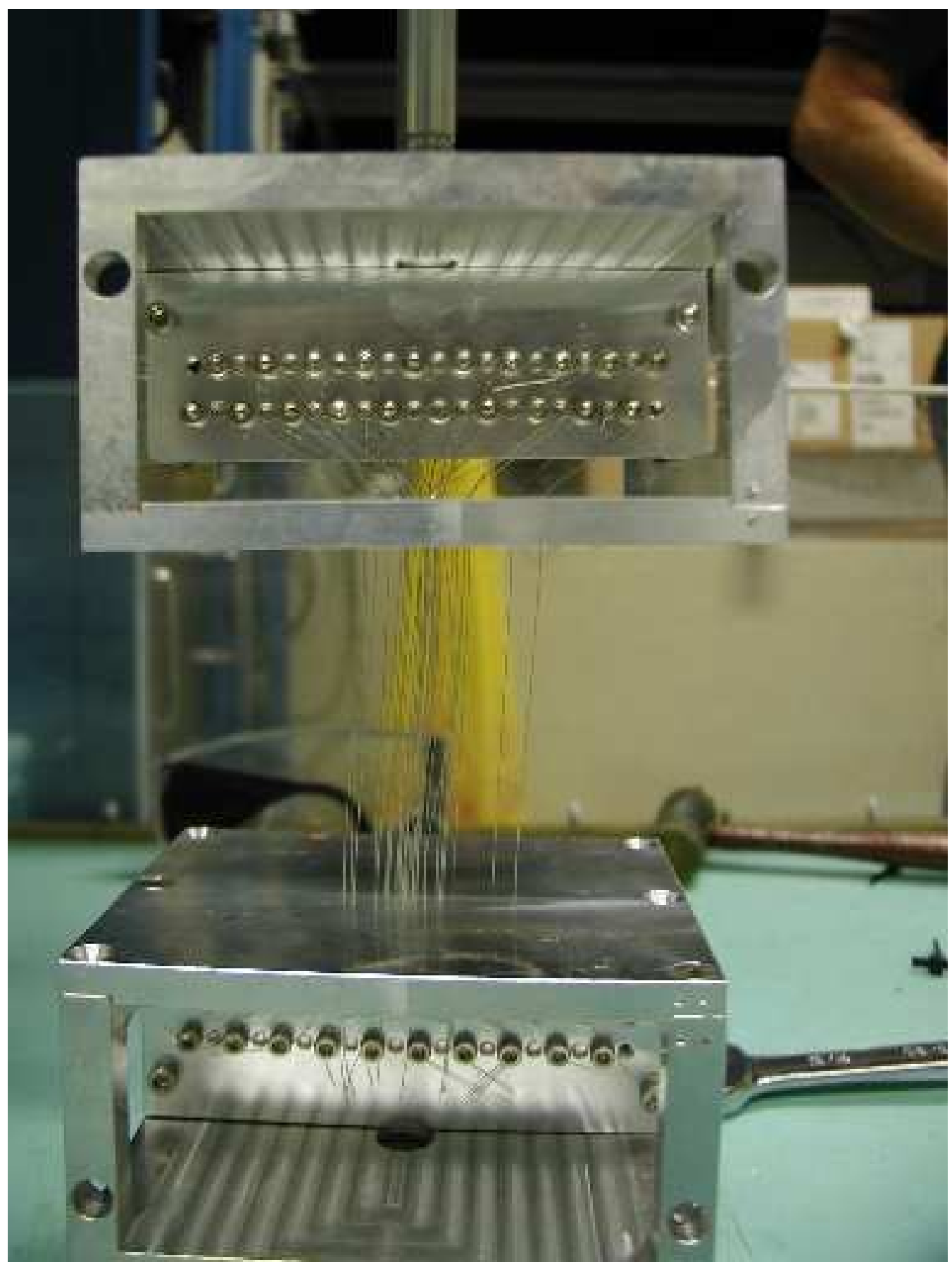

Figure 5: Detail of phantom before wires were pulled taut. 


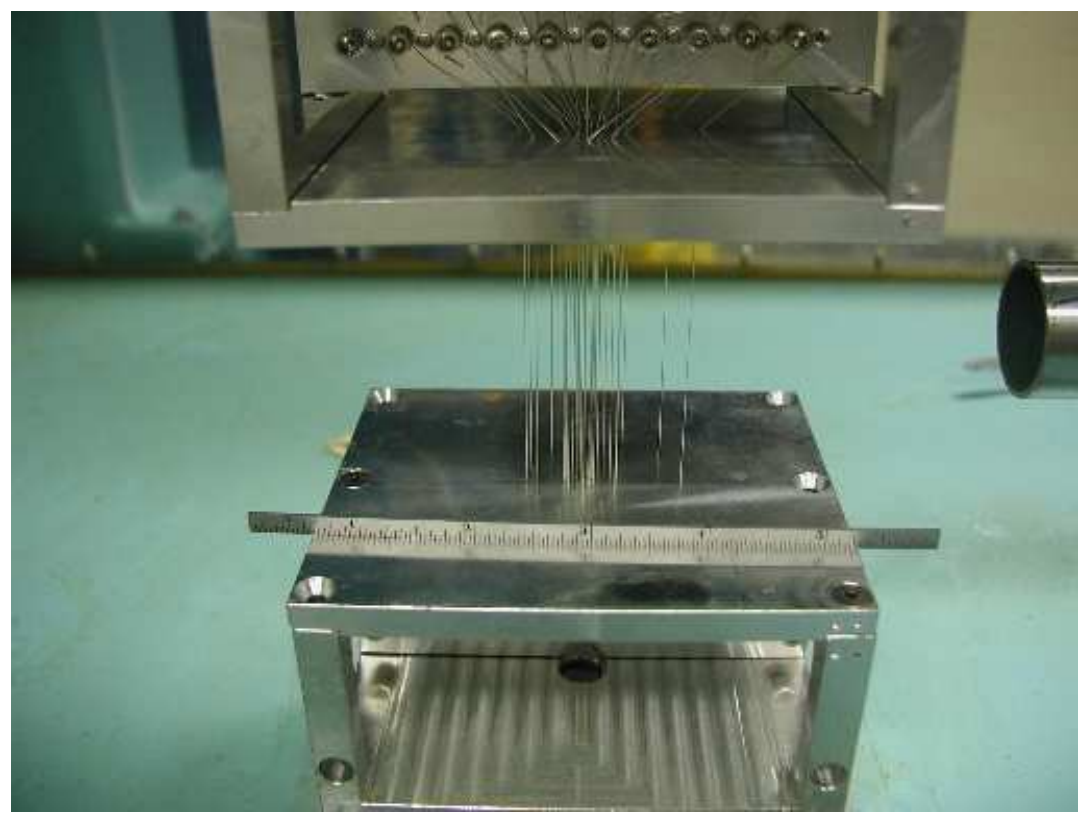

(a)

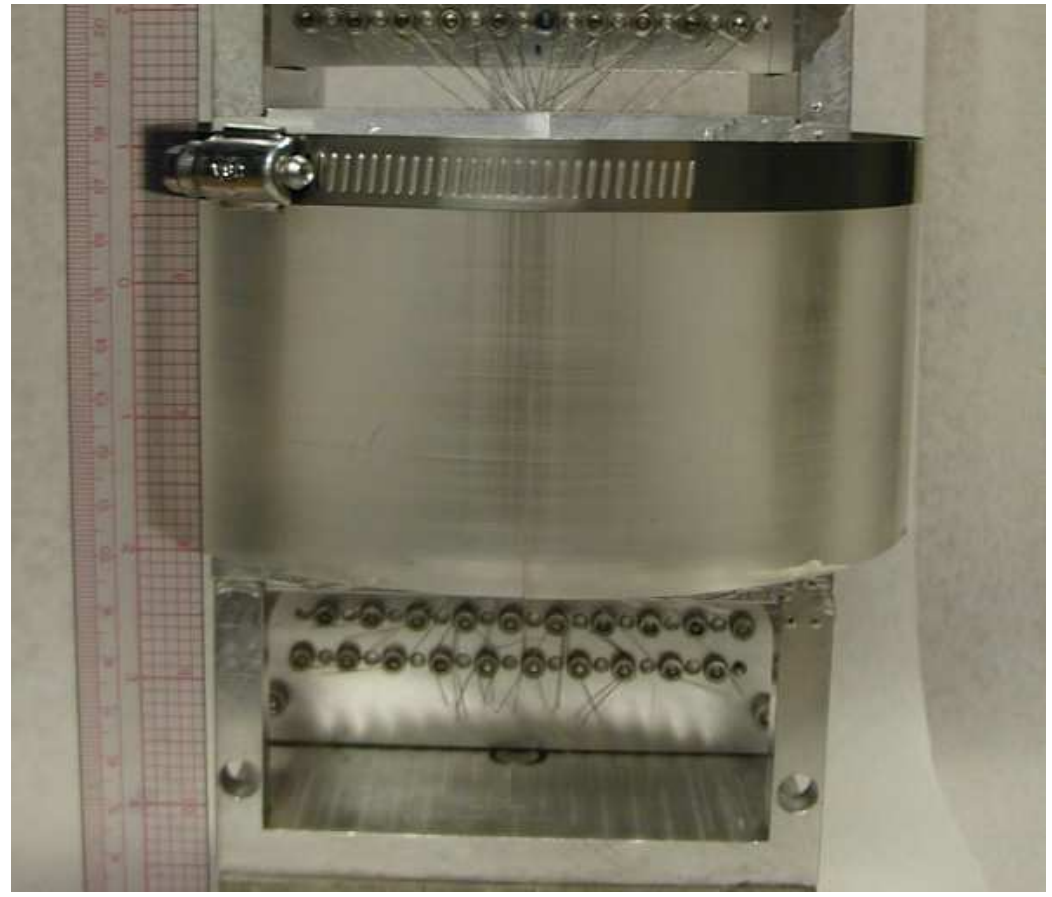

(b)

Figure 6: (a) Phantom after the wires were pulled taut. (b) Phantom with optional acrylic hollow cylinder. 


\begin{tabular}{rr|rr}
$x$ & $y$ & $x$ & $y$ \\
\hline 1.5000 & 0.0000 & -3.2365 & 5.6057 \\
1.4233 & 0.8218 & -6.1421 & 3.5462 \\
0.9004 & 1.5595 & -7.7710 & 0.0000 \\
0.0000 & 1.9731 & -7.3739 & -4.2573 \\
-1.0810 & 1.8723 & -4.6647 & -8.0795 \\
-2.0515 & 1.1844 & -0.0000 & -10.2221 \\
-2.5955 & 0.0000 & 5.6001 & -9.6997 \\
-2.4629 & -1.4219 & 10.6279 & -6.1360 \\
-1.5580 & -2.6985 & 13.4464 & -0.0000 \\
0.0000 & -3.4142 & 12.7592 & 7.3665 \\
1.8704 & -3.2397 & 8.0715 & 13.9802 \\
3.5497 & -2.0494 & 0.0000 & 17.6876 \\
4.4911 & -0.0000 & -9.6901 & 16.7837 \\
4.2615 & 2.4604 & -18.3898 & 10.6174 \\
2.6958 & 4.6693 & -23.2667 & 0.0000 \\
0.0000 & 5.9076 & &
\end{tabular}

Table 1: $(x, y)$ location of wires in millimeters for the logarithmic spiral.

- Hollow cylinder only (no wires).

Example data sets showing measured time series and spectral data are presented in Figures 7 through 10. The data are very clean and have good contrast. Clear and distinct sinograms of individual wires are seen in the steel wire data set of Figure 7.

\section{Scanner and Data Collection Details}

The LLNL ultrasonic scanner consists of two independently operating transducers which orbit about a common center. They are programmed to synthesize a fully multistatic annular array. The data sets described herein were collected using the following parameters:

- Two independently scanning transducers;

- 320 receiver locations;

- 361 transmitter locations;

- One degree increment;

- $15 \mathrm{~cm}$ radius;

- Two cycles of a sine input voltage;

- $1.6 \mathrm{MHz}$ center frequency;

- $15 \mathrm{MHz}$ sample rate;

- 4096 element time record; 

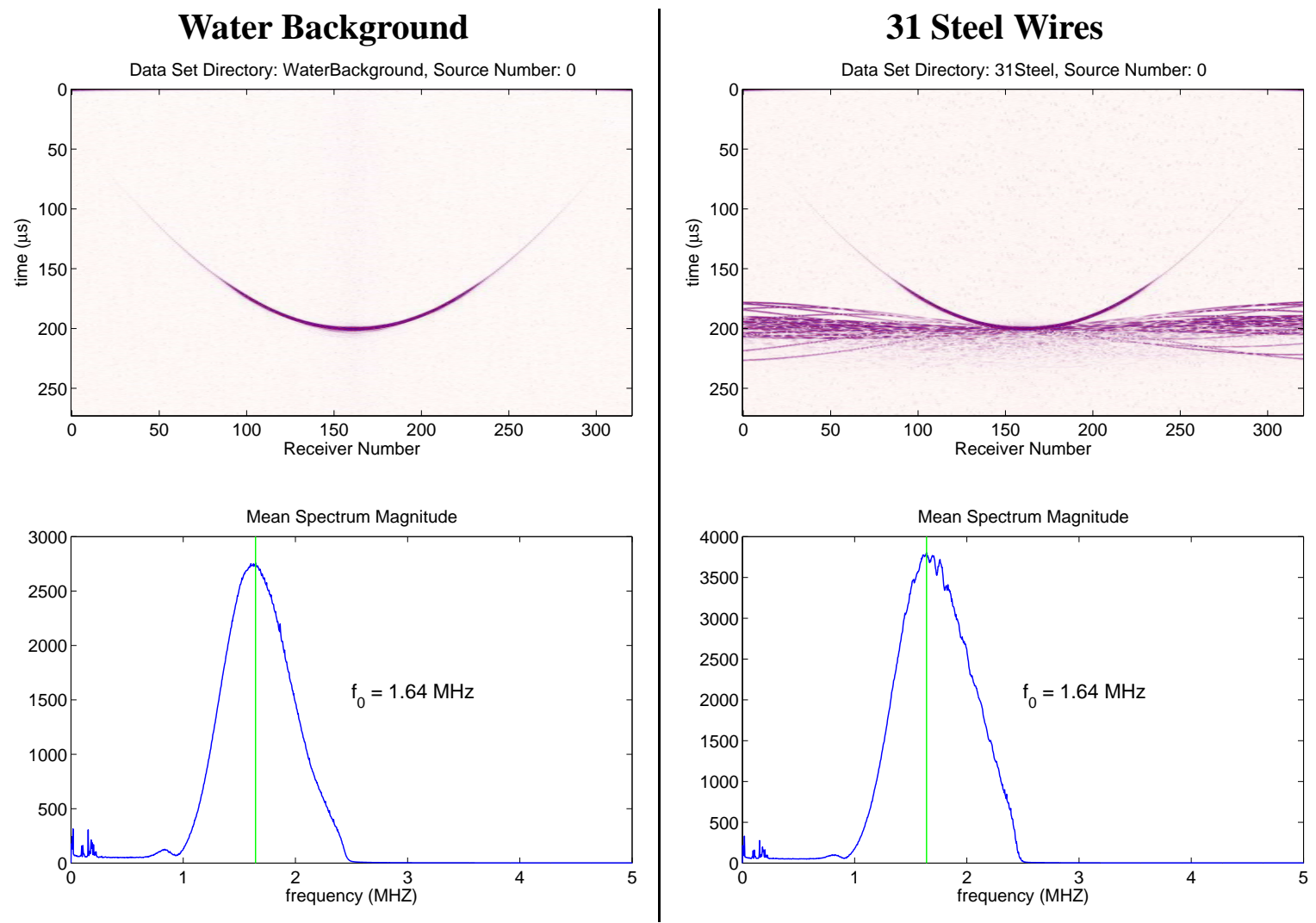

Figure 7: Example water background and 31 steel wire data sets. 

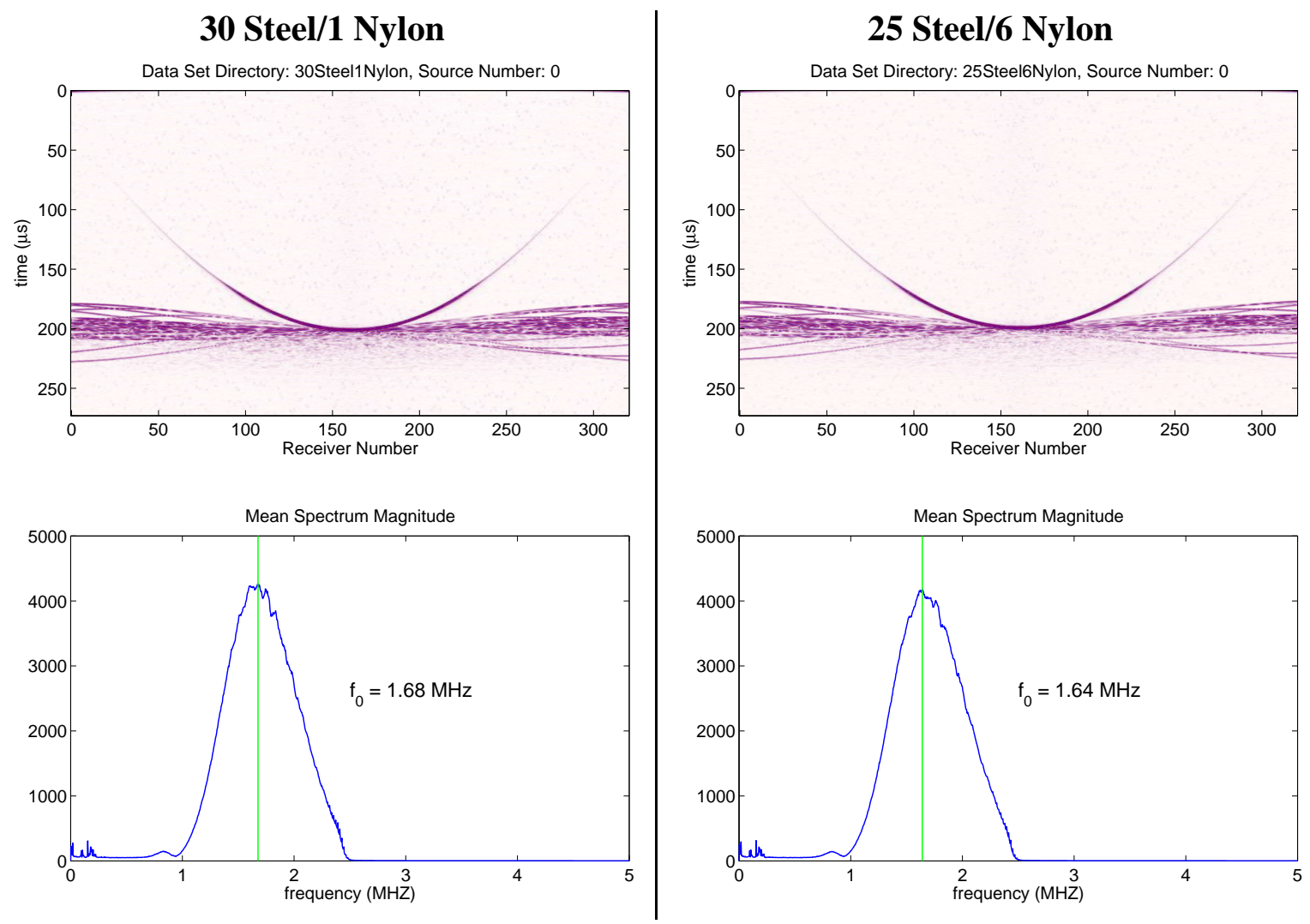

Figure 8: Example 30 steel/1 nylon and 25 steel/6 nylon data sets. 

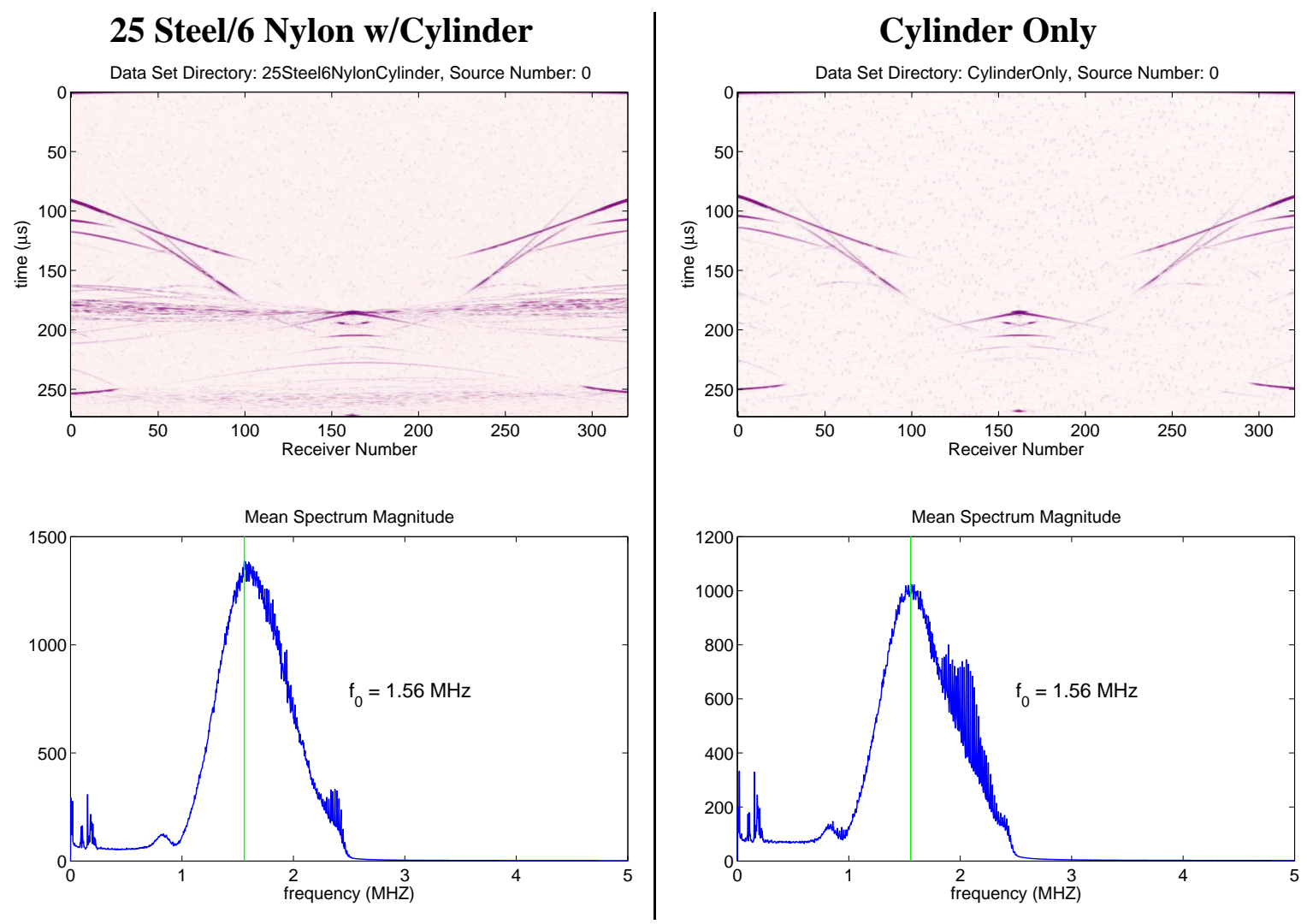

Figure 9: Example 25 steel/6 nylon and cylinder only data sets. 

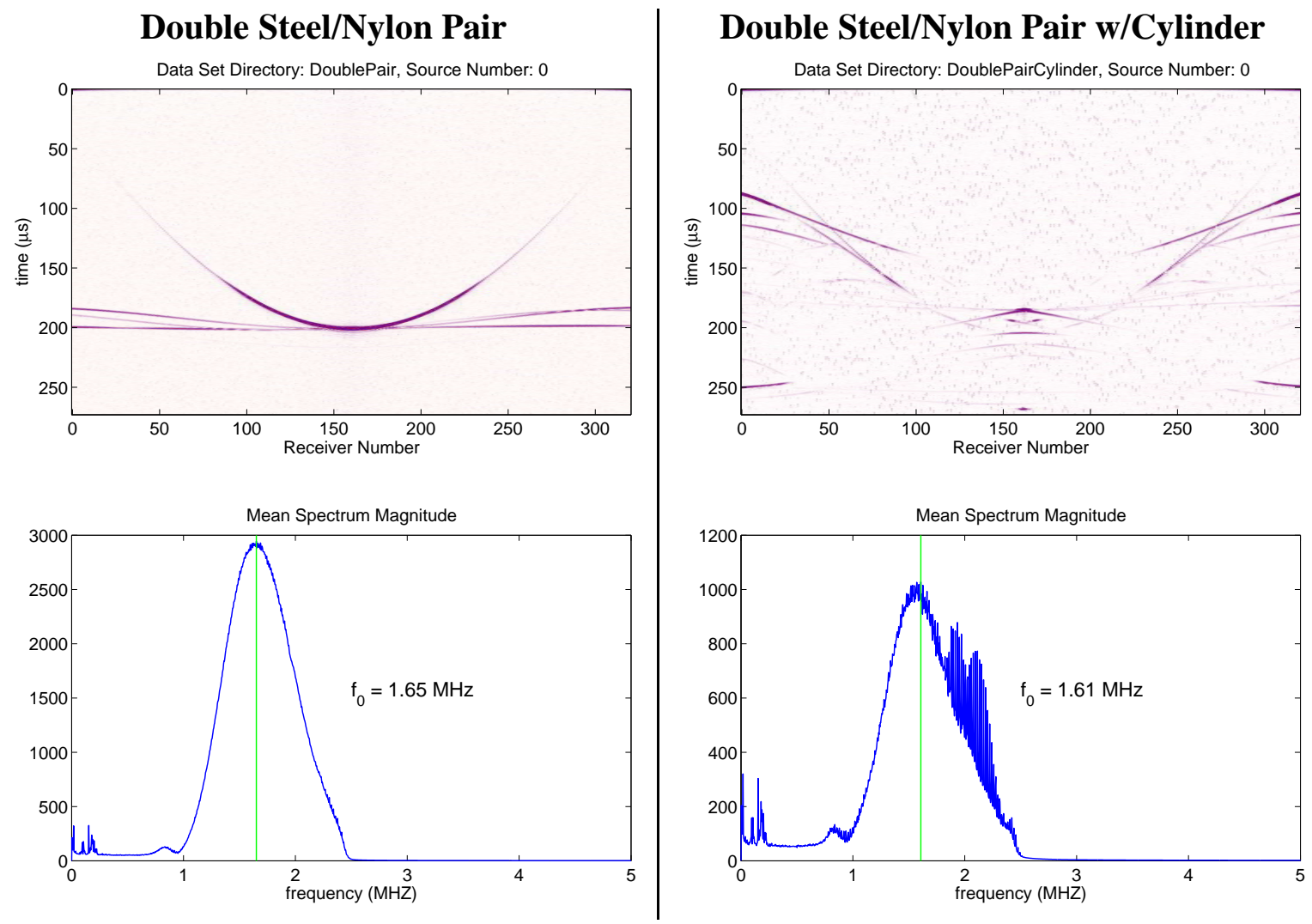

Figure 10: Example double pair data sets. 
- Multistatic data.

The complete data set for a single run are stored in a directory whose name describes the phantom configuration. For each run there are 361 text files with extension . txt which contain the metadata and a corresponding number of binary data files with extension . bin which contain the time series data. The files are labeled 000 through 360 corresponding to the index of the transmitter angular location. 


\section{MATLAB Codes to Read Data}

The following MATLAB codes are provided to read and perform a "quick look" through the data: ResolveFilename.m Used to resolve a data set and transmitter index into a full path name; flipthroughllnlusdata.m Provides a "quick look" through an entire data run; hdr2param. m Converts a raw metadata header into a header suitable for use in a reconstruction algorithm;

rdllnlusdata.m Reads one or more transmitter data sets;

rdllnlusheader.m Reads a single header file.

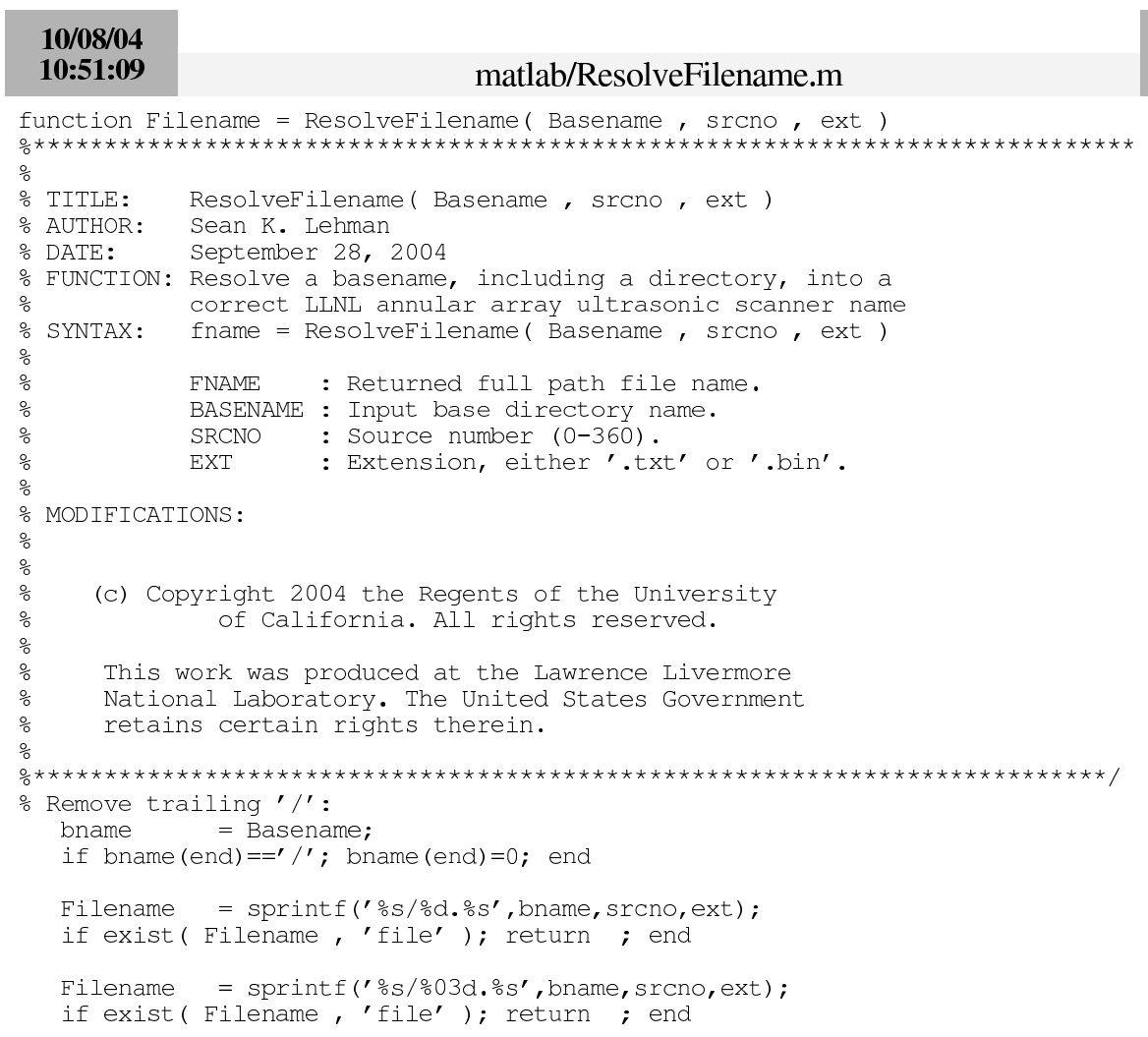




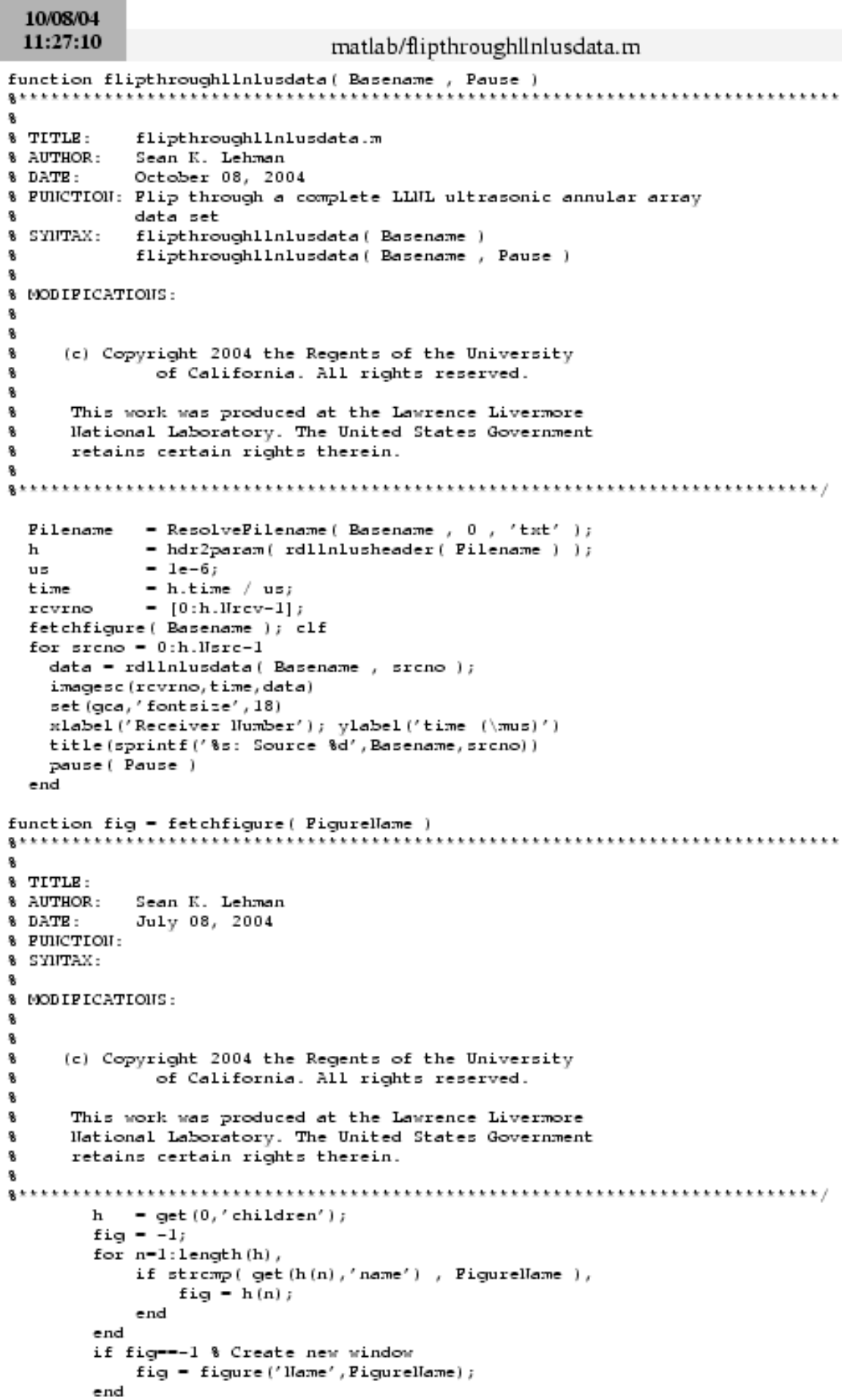


10/08/04

11:27:10

figure ( $f i g)$ matlab/flipthroughllnlusdata.m 


\section{0/08/04 \\ 10:47:10}

function param - hdr2param ( header )

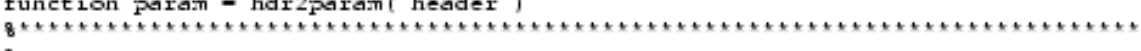
8

8 TITLE: hdr2param.en

8 AUTHOR: Sean K. Lehman

8 DATE: January 30,2003

8 pUICTIOI: Convert a raw LLIL annular array ultrazonic zcanner

header ztructure az read from the tut file into a

paraneter ztructure with information uzeful for

processing the data.

SYITAX: param - hdr2paran ( header)

PARAN : Returned parameter structure:

lircv: llumber of receiver:

lierc: liumber of zourcez

lit: llumber of time ztepz

$\mathrm{dt}$ : Time sample interval

time: Time ztep array

derc: Source angular increment

zrc0: Initial zource angluar location

drev: Receiver angular increment

rev0: Initial receiver angluar location

Radiuz: Array radiuz in $\pi n$

ercarg: Array of zource angular locationz

Rerc: Cartezian source $(x, y)$ locations

revarg: Array of receiver angular locations

Rrcv: Cartezian receiver $(x, y)$ locations

HEADER : Input rav header sturcture az returned by MODIPICATIOIS : rdllnluzheader.m or rdllnluzdata.m

(c) Copyright 2003 the Regent $z$ of the Univerzity of California. All rightz rezerved.

Thiz work waz produced at the Lavrence Livernore

llational Laboratory. The United States Government

retains certain rights therein.

$\mathrm{MH}=\quad-1=6$

$\mathrm{HH}=\quad-1 . \mathrm{e} ;$

$\begin{array}{ll}\mathrm{cm} & -1 . e-2 ; \\ \pi & -1 . e-3 ;\end{array}$

$\mathrm{nz} \quad-1 . e-3$

u $\quad-1 . e-6$

rad $\quad-\mathrm{pi} / 180$;

Irev - header.RuPositions;

liere - header.TrPositions;

lit - header.RsTimeSamplez;

dt $\quad-1 /$ (header.RsSampPreg * $(\mathrm{HH}=$ );

time $\quad-[0: 1 \mathrm{tt}-1]^{*} \mathrm{dt}$;

rad $\quad$ pi 180 ;

derc - header.Trincr * rad

erc0 - (header.TuThetaStart header.TrThetaPos) * rad;

drev - header.RoIncr * rad

rev0 - (header.RxThetaStart header.TsThetaPoz) * rad;

Radius - header.DeviceRadiuzl- * $\pi \mathrm{m}$

arg - $\operatorname{arc0}+[0: 1 \mathrm{Isc}-1]^{\prime+d a r c ;}$

Rerc - Radiuz * [ $\cos$ (arg) zin (arg) ];

arg - rev0 + [0:1Irev-1]'*drev;

Rrcv - Radiuz * [ $\cos (a r g)$ zin (arg) ];

param - etruct ('Irev', Irev, ...

'Herc', Herc, - .

'lit', lit , ... 
$10 / 08 / 04$

10:47:10

matlab/hdr2param.m

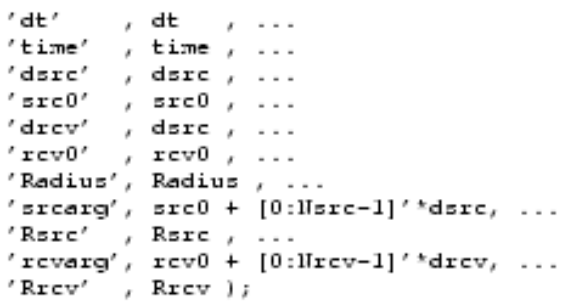




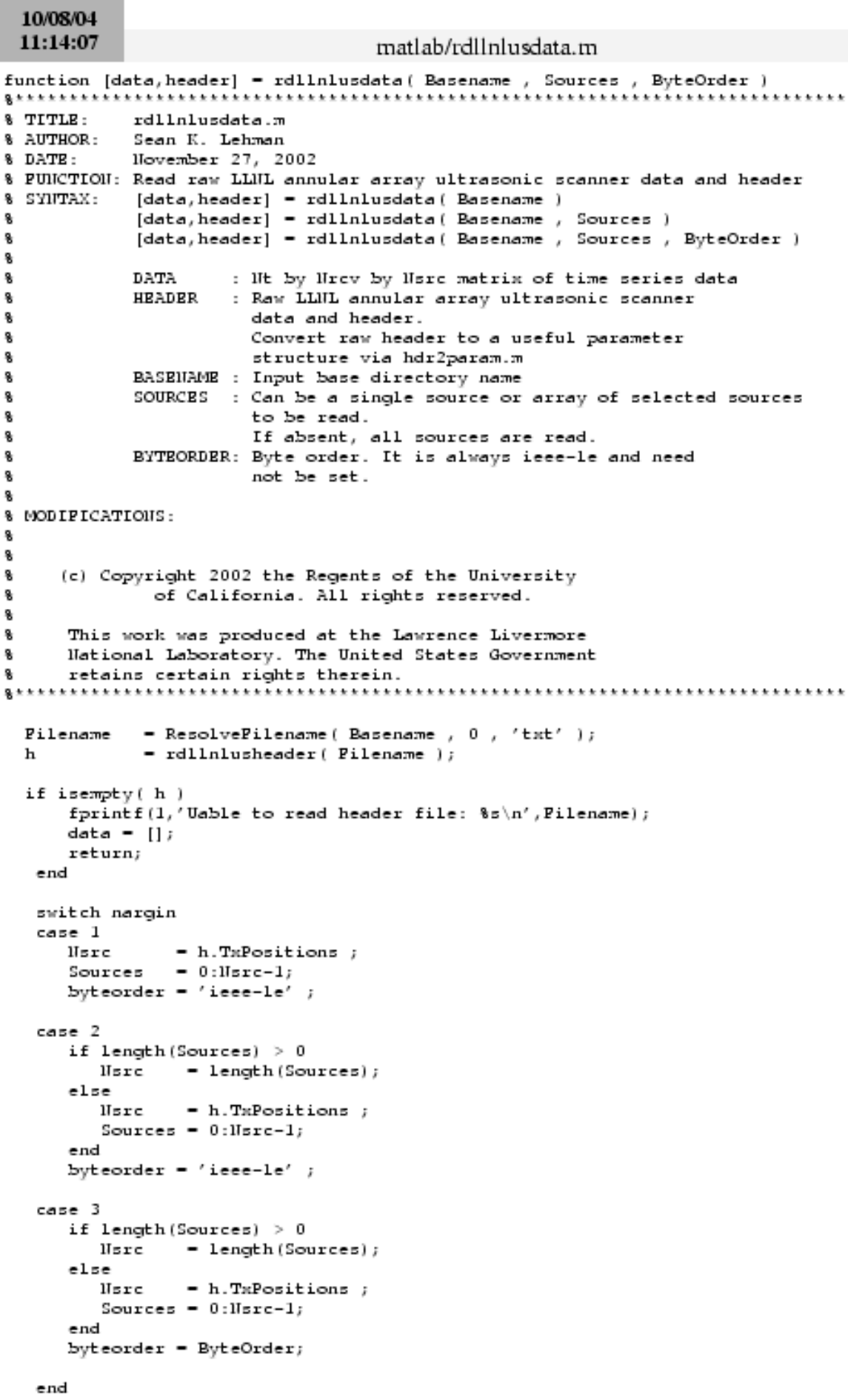




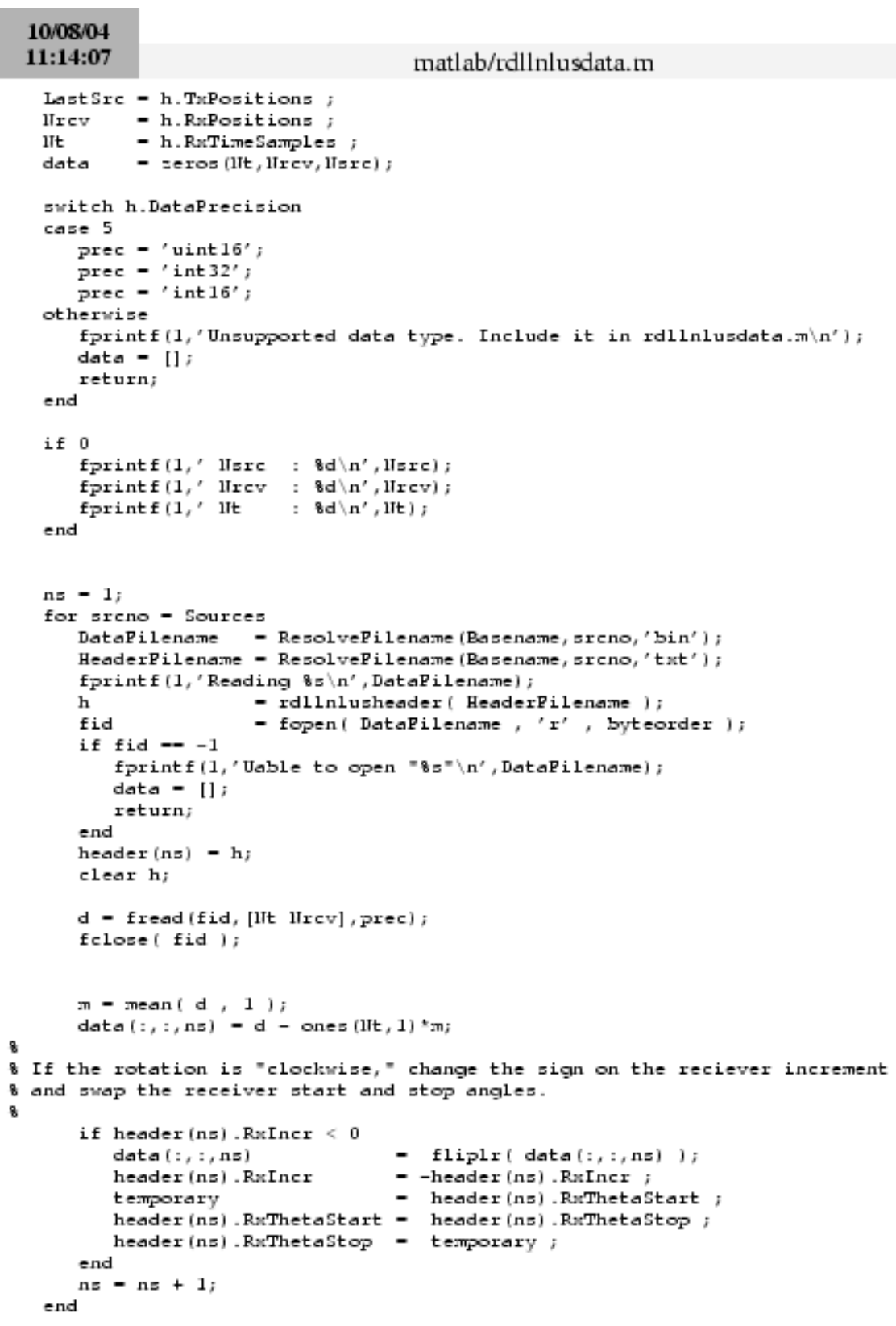




\section{0/08/04}

11:15:13

matlab/rdllnlusheader.m

function header - rdllnluzheader ( Pilename)

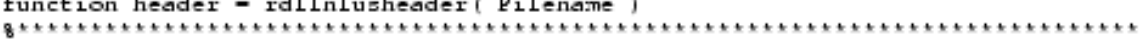

8

8 TITLE: rdllnluzheader.m

8 AUTHOR: Sean K. Lehman

8 DATE: lovember 27, 2002

8 PUICTIOI: Read raw LLIL annular array ultrazonic zcanner header

8 Sritax: Header - rdllnluzheader ( Pilename)

8 HEADER : Raw LLIIL annular array ultrazonic zcanner

data and header.

Convert raw header to a uzeful parameter

structure via hdr2paran.m

PILEIIAME : The full file name, including the extenzion, of the header file.

WODIPICATIOIS :

(c) Copyright 2002 the Regentz of the Univerzity of California. All rightz rezerved.

Thiz work was produced at the Lawrence Livermore

llational Laboratory. The United States Government

retainz certain rightz therein.

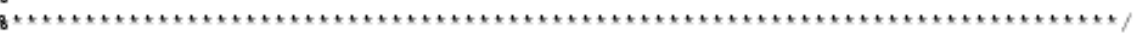

fid - fopen ( Pilename, ' $r$ ' )

if fid - - -

fprintf (1, Uable to open " $8=" \backslash \mathrm{n}^{\prime}, \mathrm{P}$ ilename);

header - []

end

return; ) ;

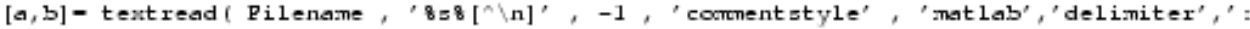

fcloze( fid);

lla - length (a);

for $n-1: 11 a$

$=\quad-\operatorname{char}(a(n))$;

- find $(=-\ldots$, ,

$\bar{z}(\mathrm{k})$;

$a\{n\}-=$;

end

header - cell2ztruct $(b, a, 1)$;

if 'izfield (header, 'BadScanLinez')

header.BadScanlinez - '0';

if 'izfield (header, 'Checl:SumBrrors')

header.CheckSumBrrorz - '0';

end

header - Convert HeaderStringz2llum ( header );

function $\mathrm{h}$ - ConvertHeaderStringz2llum ( hin )

h

h. DeviceRadiusUT - str2num (hin.DeviceRadiuzUT);

h. DeviceRadius(M - str2num (hin.DeviceRadius(J);

h.TrzPositionz - ztr2num(hin.TrzPozitions);

h.TrzPos - ztr2num(hin.TrzPos);

h.TrzStart - ztr2num(hin.TrzStart);

h.TxzStop - etr2num(hin.TxzStop); 


\begin{tabular}{|c|c|}
\hline 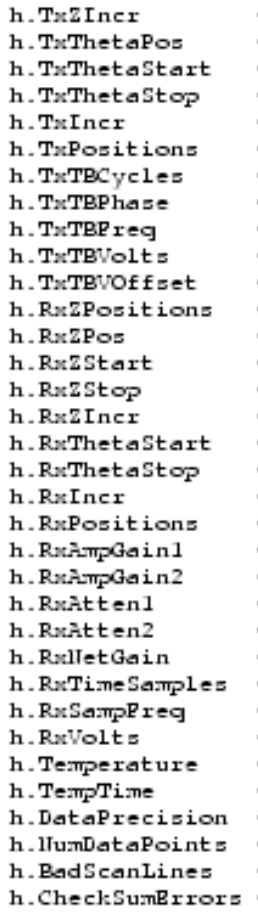 & 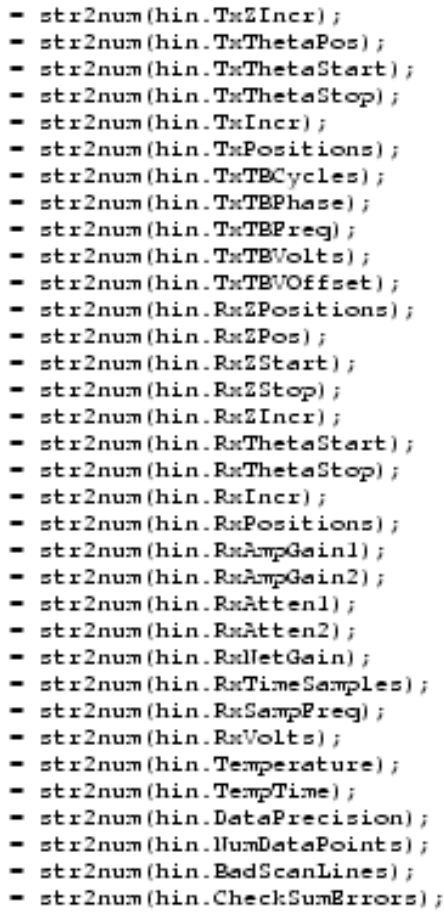 \\
\hline
\end{tabular}




\section{Typical Metadata File}

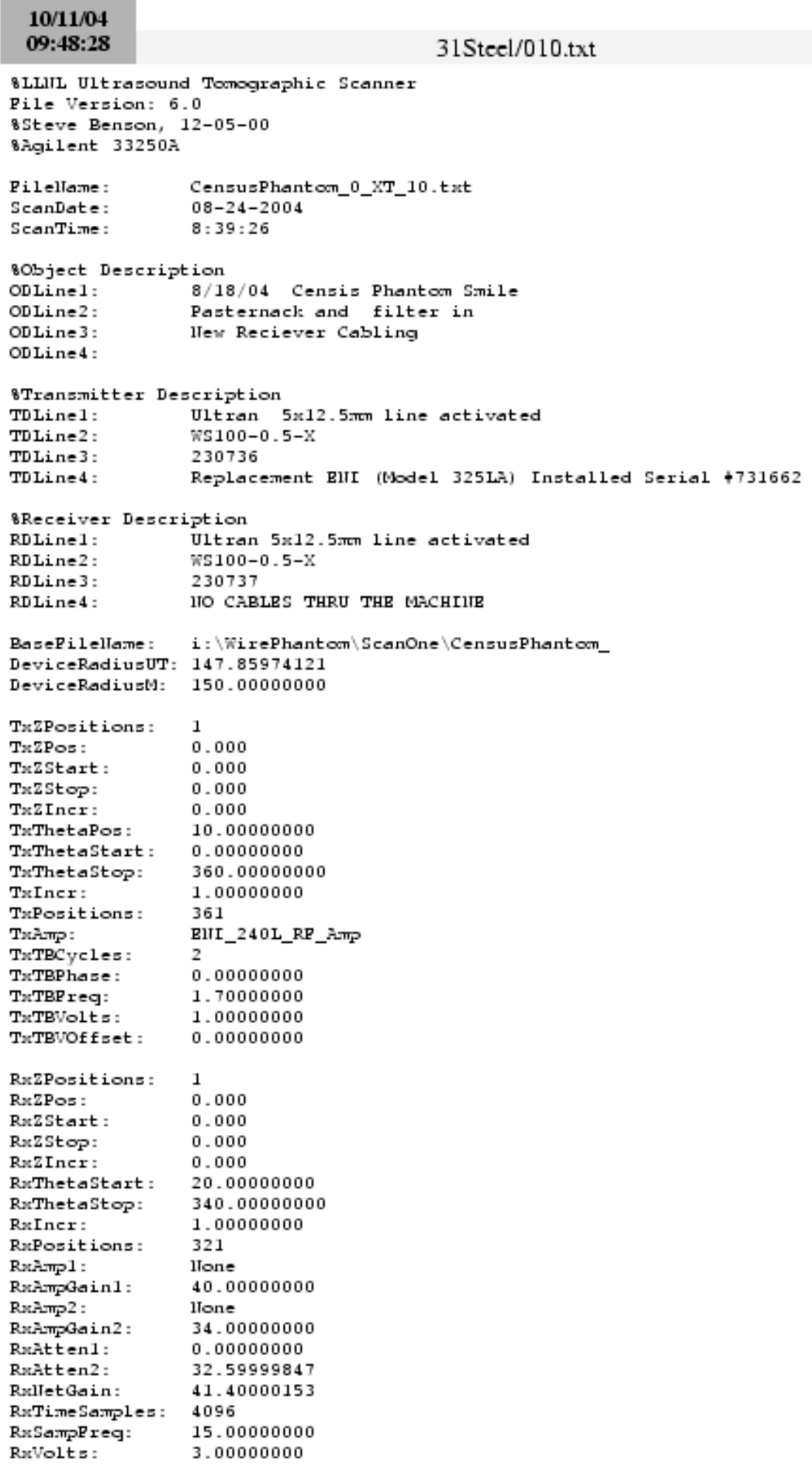


10/11/04

09:48:28

31Steel/010.txt

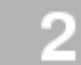

Temperature: $\quad 211.95605469$

TermpTime: $\quad 1019.15500000$

DataPrecizion: 5

llumDataPoint : : 1314816 


\section{Listing of Typical Data File}

The listing of the data set graphed here represents a typical received time series.

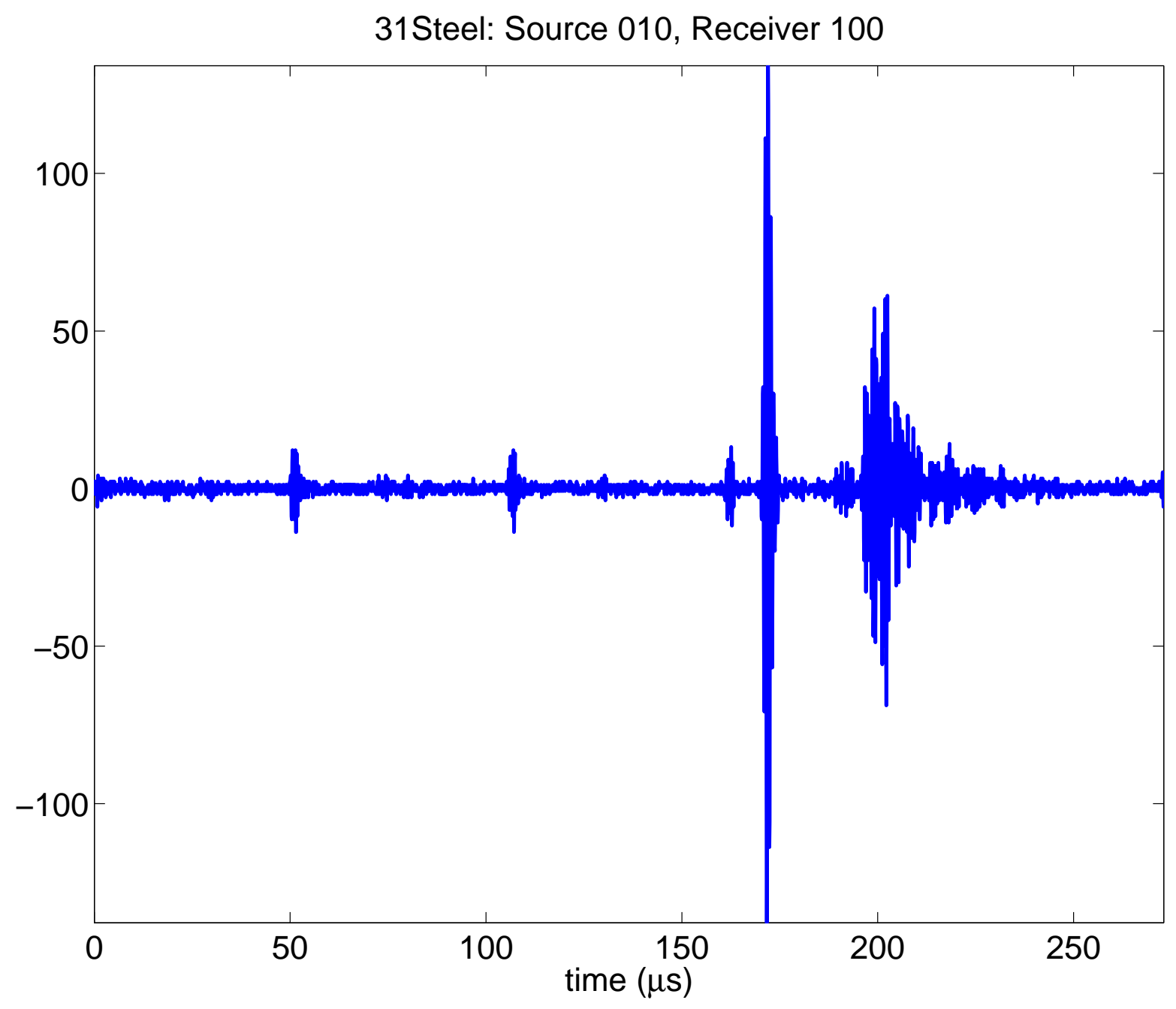




\begin{tabular}{|c|c|c|c|c|c|c|}
\hline $\begin{array}{l}10 / 11 / 04 \\
10: 09: 30\end{array}$ & & & Steel_010_ & 00.1 st & & \\
\hline 0.204834 & 2.20483 & 1.20483 & 2.20483 & -1.79517 & -1.79517 & -1.79517 \\
\hline-0.795166 & 2.20483 & -0.795166 & 2.20483 & 0.204834 & -5.79517 & -1.79517 \\
\hline 4.20483 & 0.204834 & -2.79517 & -0.795166 & 2.20483 & 3.20483 & 3.20483 \\
\hline-0.795166 & 0.204834 & 3.20483 & 0.204834 & 2.20483 & 2.20483 & -3.79517 \\
\hline-3.79517 & 2.20483 & 3.20483 & 1.20483 & -1.79517 & -0.795166 & 1.20483 \\
\hline 3.20483 & 1.20483 & -1.79517 & -1.79517 & -0.795166 & 1.20483 & 2.20483 \\
\hline-0.795166 & -1.79517 & -2.79517 & 2.20483 & 1.20483 & 1.20483 & -1.79517 \\
\hline-0.795166 & 1.20483 & 1.20483 & -0.795166 & -1.79517 & -0.795166 & 0.204834 \\
\hline 1.20483 & 1.20483 & -0.795166 & -0.795166 & 0.204834 & 2.20483 & 1.20483 \\
\hline 0.204834 & -2.79517 & -0.795166 & 1.20483 & 2.20483 & 1.20483 & 0.204834 \\
\hline 0.204834 & 0.204834 & 1.20483 & 2.20483 & 0.204834 & -0.795166 & 0.204834 \\
\hline 2.20483 & 0.204834 & -0.795166 & -1.79517 & 0.204834 & -0.795166 & 1.20483 \\
\hline 0.204834 & 0.204834 & -0.795166 & 0.204834 & 1.20483 & 0.204834 & -0.795166 \\
\hline 0.204834 & 1.20483 & 1.20483 & 1.20483 & 1.20483 & -0.795166 & 0.204834 \\
\hline 3.20483 & 0.204834 & 0.204834 & -0.795166 & -1.79517 & 0.204834 & 2.20483 \\
\hline 0.204834 & 0.204834 & 1.20483 & 1.20483 & -0.795166 & 0.204834 & 0.204834 \\
\hline 0.204834 & 0.204834 & -0.795166 & 1.20483 & -0.795166 & 0.204834 & 0.204834 \\
\hline-0.795166 & 3.20483 & 0.204834 & 0.204834 & 0.204834 & 0.204834 & 0.204834 \\
\hline 0.204834 & 1.20483 & -0.795166 & -1.79517 & 0.204834 & 0.204834 & 0.204834 \\
\hline 2.20483 & -0.795166 & 1. 20483 & 1.20483 & 1.20483 & -0.795166 & -0.795166 \\
\hline 0.204834 & 3.20483 & 0.204834 & -0.795166 & -0.795166 & 0.204834 & 0.204834 \\
\hline 0.204834 & 1.20483 & -1.79517 & -0.795166 & 1.20483 & 2.20483 & 0.204834 \\
\hline 0.204834 & -0.795166 & -1.79517 & -0.795166 & 2.20483 & 0.204834 & 0.204834 \\
\hline-0.795166 & 0.204834 & 0.204834 & 1.20483 & -0.795166 & 0.204834 & -0.795166 \\
\hline-0.795166 & 1.20483 & -0.795166 & -0.795166 & 0.204834 & 1.20483 & 0.204834 \\
\hline 1.20483 & -0.795166 & -0.795166 & -1.79517 & 2.20483 & 1.20483 & 0.204834 \\
\hline-0.795166 & -0.795166 & 0.204834 & 1.20483 & -1.79517 & -0.795166 & -0.795166 \\
\hline 0.204834 & -0.795166 & 0.204834 & 0.204834 & -2.79517 & -0.795166 & -0.795166 \\
\hline 0.204834 & -1.79517 & 0.204834 & -0.795166 & -0.795166 & -0.795166 & -0.795166 \\
\hline 0.204834 & -0.795166 & 0.204834 & 1.20483 & 0.204834 & 0.204834 & -1.79517 \\
\hline-0.795166 & -0.795166 & 1.20483 & 0.204834 & 0.204834 & 0.204834 & -0.795166 \\
\hline 0.204834 & -0.795166 & -1.79517 & 1.20483 & 2.20483 & -0.795166 & -0.795166 \\
\hline 0.204834 & -0.795166 & -1.79517 & 0.204834 & 0.204834 & 0.204834 & 0.204834 \\
\hline-0.795166 & -0.795166 & 1.20483 & 2.20483 & 0.204834 & -0.795166 & 1.20483 \\
\hline 1.20483 & -0.795166 & -0.795166 & -1.79517 & -0.795166 & -0.795166 & 1.20483 \\
\hline 0.204834 & 0.204834 & 0.204834 & -0.795166 & 0.204834 & 1.20483 & 0.204834 \\
\hline 2.20483 & 1.20483 & -1.79517 & 0.204834 & -0.795166 & -1.79517 & -0.795166 \\
\hline 1.20483 & 0.204834 & 1.20483 & -0.795166 & -0.795166 & -0.795166 & -0.795166 \\
\hline 0.204834 & 0.204834 & 1.20483 & -2.79517 & -3.79517 & 1.20483 & -0.795166 \\
\hline 0.204834 & -0.795166 & -0.795166 & 0.204834 & 0.204834 & -0.795166 & -1.79517 \\
\hline-1.79517 & 2.20483 & 1.20483 & 0.204834 & -2.79517 & -3.79517 & -0.795166 \\
\hline 1.20483 & 1.20483 & 0.204834 & -0.795166 & -1.79517 & 0.204834 & 0.204834 \\
\hline 0.204834 & -0.795166 & -0.795166 & 2.20483 & 1.20483 & 0.204834 & -1.79517 \\
\hline-0.795166 & 1.20483 & 0.204834 & 0.204834 & -1.79517 & 0.204834 & 1.20483 \\
\hline 0.204834 & 1.20483 & 0.204834 & -1.79517 & 0.204834 & 1.20483 & 1.20483 \\
\hline 2.20483 & -0.795166 & -0.795166 & 0.204834 & 2.20483 & 2.20483 & -0.795166 \\
\hline 1.20483 & -0.795166 & 0.204834 & 1.20483 & 1.20483 & -1.79517 & 0.204834 \\
\hline 1.20483 & 1.20483 & 1.20483 & 0.204834 & 0.204834 & 1.20483 & 1.20483 \\
\hline 1.20483 & -0.795166 & 0.204834 & 0.204834 & 2.20483 & 0.204834 & -0.795166 \\
\hline 0.204834 & -0.795166 & -0.795166 & 0.204834 & 0.204834 & -0.795166 & -0.795166 \\
\hline-0.795166 & 0.204834 & -0.795166 & -1.79517 & -1.79517 & -0.795166 & -1.79517 \\
\hline-0.795166 & -0.795166 & -1.79517 & -0.795166 & 0.204834 & -0.795166 & 1.20483 \\
\hline-0.795166 & -0.795166 & -0.795166 & 0.204834 & -0.795166 & -0.795166 & -1.79517 \\
\hline-0.795166 & 1.20483 & 0.204834 & -0.795166 & -0.795166 & -1.79517 & 0.204834 \\
\hline-2.79517 & 0.204834 & -0.795166 & -0.795166 & -0.795166 & 0.204834 & -1.79517 \\
\hline-0.795166 & 0.204834 & -0.795166 & 0.204834 & 0.204834 & 0.204834 & -0.795166 \\
\hline 1.20483 & 0.204834 & -0.795166 & 0.204834 & 0.204834 & 0.204834 & -0.795166 \\
\hline 0.204834 & -0.795166 & 0.204834 & -0.795166 & 1.20483 & 0.204834 & 0.204834 \\
\hline 3.20483 & 0.204834 & -1.79517 & 0.204834 & 1.20483 & 1.20483 & -0.795166 \\
\hline-0.795166 & 1.20483 & 2.20483 & 1.20483 & 0.204834 & 1.20483 & 0.204834 \\
\hline 1. 20483 & 0.204834 & -0.795166 & 1.20483 & 1. 20483 & -0.795166 & 0.204834 \\
\hline-1.79517 & 1.20483 & 0.204834 & -0.795166 & 0.204834 & 0.204834 & 2.20483 \\
\hline-0.795166 & 0.204834 & 0.204834 & -2.79517 & -1.79517 & 0.204834 & 0.204834 \\
\hline 0.204834 & -1.79517 & -2.79517 & 0.204834 & 1.20483 & 2.20483 & 0.204834 \\
\hline-2.79517 & -3.79517 & -2.79517 & -0.795166 & 2.20483 & 0.204834 & -0.795166 \\
\hline-2.79517 & -1.79517 & 1.20483 & 0.204834 & -0.795166 & -1.79517 & -1.79517 \\
\hline 0.204834 & 0.204834 & 0.204834 & -1.79517 & -1.79517 & 0.204834 & 1.20483 \\
\hline 1.20483 & -1.79517 & -1.79517 & -0.795166 & 1.20483 & 2.20483 & 0.204834 \\
\hline
\end{tabular}




\begin{tabular}{|c|c|c|c|c|c|c|}
\hline $\begin{array}{l}10 / 11 / 04 \\
10: 09: 30\end{array}$ & & & Steel_010_ & 00.1 st & & \\
\hline 0.204834 & 0.204834 & 0.204834 & 1.20483 & 1.20483 & 0.204834 & -0.795166 \\
\hline 1.20483 & 0.204834 & 1.20483 & -1.79517 & 0.204834 & 0.204834 & -0.795166 \\
\hline 0.204834 & 1.20483 & 1.20483 & 0.204834 & 0.204834 & 0.204834 & 1. 20483 \\
\hline 0.204834 & -0.795166 & 0.204834 & 0.204834 & 0.204834 & 1.20483 & 1.20483 \\
\hline 0.204834 & 1.20483 & 0.204834 & 2.20483 & -0.795166 & -0.795166 & 0.204834 \\
\hline-1.79517 & -0.795166 & 0.204834 & 0.204834 & 2.20483 & 0.204834 & -0.795166 \\
\hline-0.795166 & 0.204834 & -0.795166 & 0.204834 & 0.204834 & 1.20483 & 0.204834 \\
\hline 0.204834 & -0.795166 & -0.795166 & -0.795166 & -0.795166 & -0.795166 & 0.204834 \\
\hline 0.204834 & -0.795166 & 1. 20483 & -0.795166 & -1.79517 & -1.79517 & -0.795166 \\
\hline-0.795166 & -0.795166 & 0.204834 & -1.79517 & 0.204834 & -0.795166 & -0.795166 \\
\hline-0.795166 & 0.204834 & 0.204834 & 0.204834 & 1.20483 & 1.20483 & -1.79517 \\
\hline-0.795166 & 0.204834 & -0.795166 & 0.204834 & 0.204834 & -0.795166 & -0.795166 \\
\hline 1.20483 & 0.204834 & -0.795166 & -1.79517 & -1.79517 & -0.795166 & 0.204834 \\
\hline 0.204834 & 0.204834 & 0.204834 & 1.20483 & 1.20483 & 0.204834 & -0.795166 \\
\hline-0.795166 & -0.795166 & -0.795166 & 1.20483 & 1.20483 & -0.795166 & -0.795166 \\
\hline 0.204834 & 0.204834 & 1.20483 & -0.795166 & 1.20483 & 1. 20483 & -0.795166 \\
\hline 0.204834 & 0.204834 & -1.79517 & 1.20483 & 2.20483 & 0.204834 & -0.795166 \\
\hline-0.795166 & 1.20483 & 0.204834 & 1.20483 & 1.20483 & 0.204834 & 1.20483 \\
\hline-0.795166 & 1.20483 & 0.204834 & -0.795166 & 0.204834 & 0.204834 & 0.204834 \\
\hline-0.795166 & 1.20483 & 0.204834 & 1.20483 & 0.204834 & 1.20483 & 1.20483 \\
\hline 0.204834 & 1.20483 & -0.795166 & 0.204834 & -0.795166 & -1.79517 & 1.20483 \\
\hline 1.20483 & 0.204834 & 2.20483 & -0.795166 & -0.795166 & 0.204834 & -0.795166 \\
\hline 1.20483 & 1.20483 & -0.795166 & 0.204834 & -0.795166 & 0.204834 & 0.204834 \\
\hline 0.204834 & 0.204834 & 1.20483 & -0.795166 & 0.204834 & -0.795166 & 0.204834 \\
\hline 0.204834 & 0.204834 & 1.20483 & 0.204834 & 0.204834 & -0.795166 & -0.795166 \\
\hline 1.20483 & 1.20483 & 0.204834 & -0.795166 & -0.795166 & 1. 20483 & 1. 20483 \\
\hline-0.795166 & 0.204834 & -0.795166 & 1.20483 & 2.20483 & -0.795166 & 0.204834 \\
\hline 1.20483 & -0.795166 & -0.795166 & 1.20483 & 1.20483 & 0.204834 & 0.204834 \\
\hline-0.795166 & 1.20483 & 0.204834 & -0.795166 & 0.204834 & 0.204834 & -0.795166 \\
\hline 1.20483 & 1.20483 & -0.795166 & -0.795166 & 0.204834 & 0.204834 & 0.204834 \\
\hline 0.204834 & 0.204834 & -0.795166 & 0.204834 & -0.795166 & 0.204834 & 0.204834 \\
\hline 0.204834 & 0.204834 & 1. 20483 & -0.795166 & -0.795166 & -0.795166 & -0.795166 \\
\hline-1.79517 & 0.204834 & 0.204834 & -1.79517 & -0.795166 & 1. 20483 & 0.204834 \\
\hline 0.204834 & -0.795166 & -0.795166 & 1.20483 & 1.20483 & 0.204834 & 0.204834 \\
\hline-1.79517 & 0.204834 & 0.204834 & 0.204834 & -0.795166 & 0.204834 & 0.204834 \\
\hline-0.795166 & 1.20483 & 0.204834 & -1.79517 & 0.204834 & -0.795166 & 1.20483 \\
\hline 0.204834 & 0.204834 & -1.79517 & 0.204834 & 0.204834 & -0.795166 & 1.20483 \\
\hline 1.20483 & -0.795166 & 1.20483 & 0.204834 & 0.204834 & 0.204834 & 0.204834 \\
\hline 0.204834 & -0.795166 & 1.20483 & -1.79517 & -1.79517 & 0.204834 & -0.795166 \\
\hline 2.20483 & 0.204834 & 3.20483 & 4.20483 & 4. 20483 & -0.795166 & -8.79517 \\
\hline-9.79517 & -1.79517 & 8.20483 & 12.2048 & 5.20483 & -4.79517 & -6.79517 \\
\hline-2.79517 & 4.20483 & 4.20483 & -3.79517 & -9.79517 & -0.795166 & 10.2048 \\
\hline 12.2048 & 0.204834 & -13.7952 & -13.7952 & 0.204834 & 11.2048 & 10.2048 \\
\hline-1.79517 & -8.79517 & -6.79517 & 2.20483 & 7.20483 & 4. 20483 & -2.79517 \\
\hline-2.79517 & -0.795166 & 3.20483 & 4. 20483 & -1.79517 & -3.79517 & -1.79517 \\
\hline 4. 20483 & 1. 20483 & -0.795166 & -2.79517 & 0.204834 & 0.204834 & 1.20483 \\
\hline-2.79517 & -0.795166 & -0.795166 & 2.20483 & 3.20483 & -0.795166 & -2.79517 \\
\hline-0.795166 & 1.20483 & 2.20483 & 0.204834 & 0.204834 & -2.79517 & -0.795166 \\
\hline 2.20483 & 1.20483 & 1.20483 & -1.79517 & -0.795166 & 2.20483 & 2.20483 \\
\hline-0.795166 & -2.79517 & 0.204834 & 2.20483 & 1.20483 & 2.20483 & -0.795166 \\
\hline-0.795166 & 0.204834 & 0.204834 & 0.204834 & -0.795166 & 0.204834 & 0.204834 \\
\hline 1. 20483 & 0.204834 & 0.204834 & 1.20483 & -2.79517 & 1.20483 & 2.20483 \\
\hline-0.795166 & 0.204834 & -0.795166 & 0.204834 & -1.79517 & 1.20483 & 1.20483 \\
\hline-0.795166 & 0.204834 & 2. 20483 & -0.795166 & -1.79517 & -1.79517 & -0.795166 \\
\hline-0.795166 & 1.20483 & 1.20483 & -0.795166 & -1.79517 & 0.204834 & 1. 20483 \\
\hline-0.795166 & -1.79517 & -0.795166 & -0.795166 & -0.795166 & -1.79517 & -0.795166 \\
\hline-0.795166 & -0.795166 & 0.204834 & 0.204834 & -0.795166 & 0.204834 & -1.79517 \\
\hline 0.204834 & 0.204834 & -0.795166 & 0.204834 & -0.795166 & -0.795166 & -0.795166 \\
\hline-1.79517 & -0.795166 & -1.79517 & -1.79517 & 1.20483 & 0.204834 & -0.795166 \\
\hline-0.795166 & -0.795166 & 0.204834 & -0.795166 & 0.204834 & -0.795166 & 0.204834 \\
\hline 0.204834 & 0.204834 & 1.20483 & 0.204834 & 0.204834 & -1.79517 & -1.79517 \\
\hline 0.204834 & -0.795166 & 0.204834 & -0.795166 & 0.204834 & 1.20483 & 2.20483 \\
\hline 0.204834 & -0.795166 & -0.795166 & 2.20483 & 2.20483 & 0.204834 & -0.795166 \\
\hline-0.795166 & 0.204834 & 0.204834 & 0.204834 & 0.204834 & -0.795166 & 1. 20483 \\
\hline 0.204834 & -1.79517 & 1.20483 & 0.204834 & 1.20483 & -0.795166 & 0.204834 \\
\hline 0.204834 & -0.795166 & -0.795166 & 0.204834 & 1.20483 & 0.204834 & 0.204834 \\
\hline-0.795166 & 1.20483 & -0.795166 & 0.204834 & -0.795166 & 1.20483 & -0.795166 \\
\hline-0.795166 & 1.20483 & 0.204834 & -0.795166 & -1.79517 & -0.795166 & 0.204834 \\
\hline
\end{tabular}




\begin{tabular}{|c|c|c|c|c|c|c|}
\hline \multirow{2}{*}{$\begin{array}{c}10 / 11 / 04 \\
10: 09: 30 \\
0.204834\end{array}$} & \multicolumn{5}{|c|}{ 31Steel_010_100.1st } & \multirow{2}{*}{83} \\
\hline & -1.79517 & 1.20483 & 1.20483 & 0.204834 & 1.20483 & \\
\hline 0.204834 & 1.20483 & 0.204834 & 1.20483 & 1.20483 & 0.204834 & -0.795166 \\
\hline-1.79517 & 1.20483 & 1.20483 & 0.204834 & 1.20483 & -0.795166 & 1.20483 \\
\hline 1.20483 & 1.20483 & 0.204834 & -1.79517 & 1.20483 & -0.795166 & -0.795166 \\
\hline 1.20483 & -1.79517 & 0.204834 & 1.20483 & 1.20483 & 1.20483 & 1.20483 \\
\hline 0.204834 & 0.204834 & 0.204834 & 1.20483 & -0.795166 & 0.204834 & -0.795166 \\
\hline 1.20483 & 1.20483 & 0.204834 & 0.204834 & 0.204834 & 1.20483 & 1.20483 \\
\hline 0.204834 & -1.79517 & 0.204834 & 0.204834 & 0.204834 & 1.20483 & -0.795166 \\
\hline-1.79517 & -1.79517 & -0.795166 & 0.204834 & 1.20483 & -0.795166 & 0.204834 \\
\hline-1.79517 & -0.795166 & 0.204834 & -0.795166 & -0.795166 & -1.79517 & -0.795166 \\
\hline-0.795166 & -0.795166 & 1.20483 & 1.20483 & 0.204834 & 1.20483 & 0.204834 \\
\hline-1.79517 & -0.795166 & -1.79517 & -0.795166 & -0.795166 & 1.20483 & -0.795166 \\
\hline-0.795166 & 0.204834 & 0.204834 & 0.204834 & -0.795166 & 1.20483 & -1.79517 \\
\hline-0.795166 & 0.204834 & -1.79517 & -0.795166 & 0.204834 & 1.20483 & 0.204834 \\
\hline-0.795166 & -0.795166 & 2.20483 & 1.20483 & 0.204834 & 0.204834 & 1.20483 \\
\hline 1.20483 & 0.204834 & -0.795166 & -0.795166 & 1.20483 & -0.795166 & -0.795166 \\
\hline 0.204834 & 0.204834 & 1.20483 & 2.20483 & -0.795166 & 0.204834 & 0.204834 \\
\hline-0.795166 & -0.795166 & 0.204834 & 0.204834 & 0.204834 & -0.795166 & 0.204834 \\
\hline 0.204834 & -1.79517 & -0.795166 & 0.204834 & 0.204834 & 1.20483 & 2.20483 \\
\hline-0.795166 & -1.79517 & -0.795166 & -0.795166 & 4.20483 & 1.20483 & 2.20483 \\
\hline 0.204834 & -1.79517 & -0.795166 & 0.204834 & 0.204834 & 0.204834 & 1.20483 \\
\hline 0.204834 & 0.204834 & -0.795166 & 2.20483 & -0.795166 & -2.79517 & -1.79517 \\
\hline 0.204834 & -0.795166 & 0.204834 & 0.204834 & 0.204834 & 1.20483 & 0.204834 \\
\hline 1.20483 & -1.79517 & -0.795166 & 1.20483 & 4.20483 & 1.20483 & 0.204834 \\
\hline-3.79517 & 0.204834 & 1.20483 & 3.20483 & 2.20483 & -0.795166 & 0.204834 \\
\hline 1.20483 & 1.20483 & 0.204834 & 1.20483 & -0.795166 & 0.204834 & 0.204834 \\
\hline 1.20483 & 1.20483 & -1.79517 & 1.20483 & 2.20483 & 2.20483 & 1.20483 \\
\hline 0.204834 & 0.204834 & 1.20483 & 2.20483 & 0.204834 & -0.795166 & -0.795166 \\
\hline 0.204834 & 1.20483 & 2.20483 & 0.204834 & -0.795166 & -0.795166 & 1.20483 \\
\hline 0.204834 & -1.79517 & -1.79517 & 0.204834 & -0.795166 & 0.204834 & 0.204834 \\
\hline 0.204834 & -0.795166 & 0.204834 & 1.20483 & -0.795166 & 0.204834 & -0.795166 \\
\hline 0.204834 & 0.204834 & 0.204834 & -1.79517 & -0.795166 & 0.204834 & 0.204834 \\
\hline-0.795166 & -0.795166 & 1.20483 & 0.204834 & 0.204834 & -0.795166 & -0.795166 \\
\hline-0.795166 & 0.204834 & -2.79517 & -1.79517 & 1.20483 & 2.20483 & 1.20483 \\
\hline-0.795166 & -0.795166 & -0.795166 & 0.204834 & 0.204834 & 2.20483 & 0.204834 \\
\hline-2.79517 & -0.795166 & -0.795166 & 2.20483 & 4.20483 & 0.204834 & -2.79517 \\
\hline-2.79517 & -0.795166 & 1.20483 & 2.20483 & 1.20483 & 0.204834 & -0.795166 \\
\hline 0.204834 & 1.20483 & 1.20483 & -0.795166 & -2.79517 & 0.204834 & 2.20483 \\
\hline 2.20483 & -0.795166 & 0.204834 & 0.204834 & 0.204834 & 0.204834 & 0.204834 \\
\hline 0.204834 & -0.795166 & -0.795166 & 1.20483 & 1.20483 & 1.20483 & -0.795166 \\
\hline 0.204834 & -0.795166 & 1.20483 & 0.204834 & -0.795166 & -0.795166 & 0.204834 \\
\hline-0.795166 & -0.795166 & 0.204834 & 0.204834 & 0.204834 & -0.795166 & -0.795166 \\
\hline-2.79517 & 0.204834 & -0.795166 & 0.204834 & 0.204834 & -0.795166 & -1.79517 \\
\hline-0.795166 & 1.20483 & 0.204834 & 1.20483 & 0.204834 & -0.795166 & -2.79517 \\
\hline-1.79517 & -0.795166 & 0.204834 & 0.204834 & 0.204834 & 2.20483 & 0.204834 \\
\hline 0.204834 & 0.204834 & 0.204834 & -0.795166 & -0.795166 & -1.79517 & 0.204834 \\
\hline 0.204834 & 1.20483 & 2.20483 & 0.204834 & -0.795166 & 2.20483 & -0.795166 \\
\hline 0.204834 & -0.795166 & -0.795166 & 0.204834 & -0.795166 & 1.20483 & 2.20483 \\
\hline 0.204834 & -0.795166 & 0.204834 & -0.795166 & -0.795166 & 0.204834 & -1.79517 \\
\hline-0.795166 & -0.795166 & -0.795166 & 1.20483 & -0.795166 & 0.204834 & 0.204834 \\
\hline 0.204834 & -0.795166 & -1.79517 & 1.20483 & 0.204834 & 0.204834 & -0.795166 \\
\hline-0.795166 & 0.204834 & 0.204834 & -0.795166 & 0.204834 & 0.204834 & 0.204834 \\
\hline 1.20483 & 1.20483 & -0.795166 & 1.20483 & -0.795166 & -0.795166 & -1.79517 \\
\hline 0.204834 & 0.204834 & -0.795166 & -1.79517 & 1.20483 & 0.204834 & -0.795166 \\
\hline-1.79517 & 0.204834 & -0.795166 & 0.204834 & -1.79517 & -1.79517 & 0.204834 \\
\hline-1.79517 & 0.204834 & 0.204834 & 0.204834 & -0.795166 & -1.79517 & 0.204834 \\
\hline-0.795166 & 0.204834 & -0.795166 & -1.79517 & -1.79517 & 1.20483 & 0.204834 \\
\hline-0.795166 & -0.795166 & -0.795166 & -0.795166 & 0.204834 & -0.795166 & -0.795166 \\
\hline 1.20483 & -0.795166 & -0.795166 & -0.795166 & -0.795166 & -0.795166 & 1.20483 \\
\hline 0.204834 & -0.795166 & -0.795166 & 0.204834 & 0.204834 & 1.20483 & 1.20483 \\
\hline 0.204834 & -0.795166 & 0.204834 & 1.20483 & 2.20483 & 1.20483 & -1.79517 \\
\hline 1.20483 & 0.204834 & -1.79517 & -1.79517 & 0.204834 & -0.795166 & -1.79517 \\
\hline 1.20483 & 0.204834 & 0.204834 & -0.795166 & 1.20483 & 0.204834 & 0.204834 \\
\hline 0.204834 & -0.795166 & -0.795166 & 0.204834 & 2.20483 & 0.204834 & 0.204834 \\
\hline-0.795166 & 1.20483 & 1.20483 & 0.204834 & -1.79517 & -0.795166 & 1.20483 \\
\hline 1.20483 & 1.20483 & 0.204834 & 0.204834 & 0.204834 & 1.20483 & -0.795166 \\
\hline-0.795166 & -0.795166 & 0.204834 & 1.20483 & 1.20483 & 0.204834 & 1. 20483 \\
\hline 0.204834 & -0.795166 & 0.204834 & 1.20483 & 0.204834 & -1.79517 & 0.204834 \\
\hline
\end{tabular}




\begin{tabular}{|c|c|c|c|c|c|c|}
\hline $\begin{array}{l}10 / 11 / 04 \\
10: 09: 30\end{array}$ & & & Steel_010_ & $0.1 s t$ & & \\
\hline 0.204834 & 1.20483 & -1.79517 & -0.795166 & 0.204834 & 0.204834 & -0.795166 \\
\hline-0.795166 & -0.795166 & -0.795166 & 0.204834 & -0.795166 & 1.20483 & -0.795166 \\
\hline-1.79517 & -0.795166 & 0.204834 & -0.795166 & 0.204834 & 0.204834 & -1.79517 \\
\hline-0.795166 & 0.204834 & 3.20483 & -0.795166 & -0.795166 & -1.79517 & 0.204834 \\
\hline-0.795166 & 0.204834 & 0.204834 & -0.795166 & 0.204834 & 1.20483 & 0.204834 \\
\hline-1.79517 & -0.795166 & 0.204834 & -0.795166 & -0.795166 & -0.795166 & -0.795166 \\
\hline-0.795166 & 0.204834 & -0.795166 & -0.795166 & -0.795166 & 0.204834 & -1.79517 \\
\hline 0.204834 & 1.20483 & -0.795166 & -0.795166 & -0.795166 & 0.204834 & 1.20483 \\
\hline 1.20483 & 0.204834 & -0.795166 & 0.204834 & 0.204834 & 0.204834 & 0.204834 \\
\hline-1.79517 & -0.795166 & 2.20483 & 1.20483 & 0.204834 & -0.795166 & 0.204834 \\
\hline 0.204834 & 1.20483 & 0.204834 & -0.795166 & 0.204834 & 0.204834 & 1.20483 \\
\hline 0.204834 & 0.204834 & 0.204834 & -0.795166 & 0.204834 & 0.204834 & 1.20483 \\
\hline-0.795166 & 1.20483 & -0.795166 & 1.20483 & 0.204834 & 0.204834 & 0.204834 \\
\hline 0.204834 & 0.204834 & 0.204834 & 0.204834 & 1.20483 & 0.204834 & 0.204834 \\
\hline-0.795166 & 0.204834 & 0.204834 & 0.204834 & 2.20483 & 0.204834 & 0.204834 \\
\hline 0.204834 & -0.795166 & 1.20483 & -0.795166 & -1.79517 & 0.204834 & -0.795166 \\
\hline 1.20483 & 0.204834 & 0.204834 & -0.795166 & -0.795166 & 0.204834 & 1.20483 \\
\hline 0.204834 & 1.20483 & 1.20483 & -0.795166 & 0.204834 & 0.204834 & 1.20483 \\
\hline 0.204834 & 0.204834 & 1.20483 & 1.20483 & -0.795166 & 0.204834 & -0.795166 \\
\hline 1.20483 & 1.20483 & 0.204834 & 0.204834 & 0.204834 & 1.20483 & 1.20483 \\
\hline 0.204834 & 0.204834 & -0.795166 & 0.204834 & 0.204834 & -0.795166 & 1.20483 \\
\hline 0.204834 & 0.204834 & 1.20483 & 0.204834 & 2.20483 & -1.79517 & 1.20483 \\
\hline-0.795166 & -0.795166 & -1.79517 & 1.20483 & 2.20483 & 6.20483 & 0.204834 \\
\hline-2.79517 & -6.79517 & -6.79517 & -0.795166 & 7.20483 & 10.2048 & 2.20483 \\
\hline-5.79517 & -6.79517 & 0.204834 & 3.20483 & 4.20483 & -5.79517 & -8.79517 \\
\hline-0.795166 & 11.2048 & 12.2048 & 1.20483 & -13.7952 & -12.7952 & -0.795166 \\
\hline 8.20483 & 11.2048 & -0.795166 & -6.79517 & -5.79517 & -0.795166 & 3.20483 \\
\hline 2.20483 & -1.79517 & 0.204834 & 3.20483 & 1.20483 & -1.79517 & -4.79517 \\
\hline-1.79517 & 2.20483 & 4.20483 & 1.20483 & -1.79517 & -2.79517 & 1.20483 \\
\hline 3.20483 & 1.20483 & 0.204834 & -0.795166 & -0.795166 & 1.20483 & 1.20483 \\
\hline-0.795166 & -1.79517 & -0.795166 & 1. 20483 & 1. 20483 & 0.204834 & -2.79517 \\
\hline-0.795166 & 2.20483 & 3. 20483 & 1.20483 & -0.795166 & -0.795166 & 0.204834 \\
\hline 0.204834 & 1.20483 & 0.204834 & -0.795166 & -1.79517 & 1. 20483 & 1.20483 \\
\hline 0.204834 & -0.795166 & -0.795166 & 1. 20483 & 1.20483 & -0.795166 & -1.79517 \\
\hline-0.795166 & 0.204834 & 1.20483 & 1.20483 & -0.795166 & -1.79517 & -1.79517 \\
\hline 0.204834 & 1.20483 & 2.20483 & -1.79517 & -1.79517 & 0.204834 & 1.20483 \\
\hline 1.20483 & -0.795166 & -0.795166 & 1.20483 & 0.204834 & -0.795166 & 0.204834 \\
\hline 1.20483 & 1. 20483 & 0.204834 & 0.204834 & 2.20483 & 0.204834 & 0.204834 \\
\hline 1. 20483 & 0.204834 & 0.204834 & -0.795166 & -0.795166 & 1.20483 & -0.795166 \\
\hline 0.204834 & 0.204834 & 0.204834 & 0.204834 & 0.204834 & 0.204834 & 0.204834 \\
\hline 0.204834 & -0.795166 & 0.204834 & 1.20483 & -0.795166 & -0.795166 & -2.79517 \\
\hline 0.204834 & 0.204834 & 0.204834 & 0.204834 & 0.204834 & 0.204834 & -1.79517 \\
\hline 1.20483 & 0.204834 & 0.204834 & 0.204834 & 0.204834 & -1.79517 & 0.204834 \\
\hline-0.795166 & -1.79517 & -0.795166 & -0.795166 & 1.20483 & 0.204834 & 0.204834 \\
\hline-0.795166 & 0.204834 & -0.795166 & 0.204834 & -0.795166 & 1.20483 & -0.795166 \\
\hline-0.795166 & -1.79517 & 0.204834 & -0.795166 & 0.204834 & 0.204834 & 0.204834 \\
\hline-0.795166 & 0.204834 & 0.204834 & -0.795166 & -0.795166 & 1.20483 & 1.20483 \\
\hline-0.795166 & -0.795166 & 1.20483 & 0.204834 & 1.20483 & 1.20483 & -0.795166 \\
\hline 0.204834 & -0.795166 & -0.795166 & 2.20483 & 0.204834 & -0.795166 & 1. 20483 \\
\hline 1.20483 & 0.204834 & 0.204834 & 0.204834 & 1.20483 & 0.204834 & 1.20483 \\
\hline-0.795166 & -0.795166 & 0.204834 & 2.20483 & 0.204834 & 0.204834 & -0.795166 \\
\hline 1. 20483 & 1. 20483 & 0.204834 & 0.204834 & 0.204834 & -0.795166 & 2.20483 \\
\hline 0.204834 & 2.20483 & 1.20483 & 0.204834 & -0.795166 & 1.20483 & 2.20483 \\
\hline 1.20483 & 0.204834 & 0.204834 & 0.204834 & -0.795166 & 1.20483 & 1.20483 \\
\hline 0.204834 & 0.204834 & 0.204834 & 0.204834 & 0.204834 & 0.204834 & 0.204834 \\
\hline 0.204834 & 1.20483 & 0.204834 & -0.795166 & -0.795166 & -0.795166 & -0.795166 \\
\hline 2.20483 & 1.20483 & 0.204834 & -0.795166 & 2.20483 & 0.204834 & 1.20483 \\
\hline 0.204834 & 1.20483 & 0.204834 & 1. 20483 & 1.20483 & 1.20483 & -0.795166 \\
\hline-0.795166 & -1.79517 & -1.79517 & -0.795166 & 0.204834 & -0.795166 & 0.204834 \\
\hline 0.204834 & 0.204834 & -0.795166 & -0.795166 & 1.20483 & -0.795166 & 2.20483 \\
\hline 0.204834 & -0.795166 & 0.204834 & -0.795166 & 1.20483 & -1.79517 & -0.795166 \\
\hline 0.204834 & -0.795166 & 0.204834 & -0.795166 & -0.795166 & -1.79517 & -0.795166 \\
\hline 0.204834 & 0.204834 & -0.795166 & -0.795166 & -1.79517 & 0.204834 & -0.795166 \\
\hline 0.204834 & 0.204834 & 0.204834 & 1. 20483 & 0.204834 & -0.795166 & 0.204834 \\
\hline-1.79517 & -0.795166 & 0.204834 & 0.204834 & -0.795166 & 0.204834 & 0.204834 \\
\hline 0.204834 & 0.204834 & 0.204834 & -0.795166 & -0.795166 & -0.795166 & 2.20483 \\
\hline 0.204834 & -1.79517 & 1.20483 & 0.204834 & 0.204834 & 0.204834 & 0.204834 \\
\hline 0.204834 & -0.795166 & -0.795166 & 1.20483 & 2.20483 & -0.795166 & 0.204834 \\
\hline
\end{tabular}




\begin{tabular}{|c|c|c|c|c|c|c|}
\hline $\begin{array}{l}10 / 11 / 04 \\
10: 09: 30\end{array}$ & & & Steel_010_ & $00.1 s t$ & & \\
\hline 2.20483 & 0.204834 & 0.204834 & -0.795166 & -0.795166 & 0.204834 & 1.20483 \\
\hline 0.204834 & -0.795166 & -0.795166 & -0.795166 & -0.795166 & 0.204834 & 1.20483 \\
\hline 0.204834 & -0.795166 & 0.204834 & -0.795166 & 1.20483 & -0.795166 & -0.795166 \\
\hline-0.795166 & -0.795166 & 1.20483 & 0.204834 & -0.795166 & -2.79517 & 2.20483 \\
\hline 1.20483 & 1.20483 & -0.795166 & 0.204834 & -1.79517 & 0.204834 & -0.795166 \\
\hline 0.204834 & -0.795166 & -1.79517 & -0.795166 & 3.20483 & 1.20483 & 1.20483 \\
\hline-2.79517 & 0.204834 & 2.20483 & 0.204834 & 0.204834 & -0.795166 & -0.795166 \\
\hline 2.20483 & 4.20483 & 4.20483 & 1.20483 & -3.79517 & -2.79517 & 0.204834 \\
\hline 3.20483 & 1.20483 & 0.204834 & 0.204834 & 1.20483 & 1.20483 & -0.795166 \\
\hline-0.795166 & 1.20483 & 0.204834 & -0.795166 & 0.204834 & -0.795166 & 0.204834 \\
\hline 1.20483 & 0.204834 & 0.204834 & -0.795166 & -1.79517 & 0.204834 & 1.20483 \\
\hline 0.204834 & -0.795166 & -0.795166 & 0.204834 & 0.204834 & 0.204834 & -0.795166 \\
\hline-0.795166 & -0.795166 & 1.20483 & 0.204834 & 0.204834 & 0.204834 & 0.204834 \\
\hline 0.204834 & -1.79517 & 0.204834 & 0.204834 & -2.79517 & 1.20483 & 1.20483 \\
\hline 0.204834 & 0.204834 & 0.204834 & 0.204834 & 1.20483 & 0.204834 & 1.20483 \\
\hline-1.79517 & 0.204834 & -0.795166 & 1.20483 & 1.20483 & 1.20483 & -0.795166 \\
\hline-1.79517 & -0.795166 & -0.795166 & 0.204834 & 0.204834 & -1.79517 & 1.20483 \\
\hline 0.204834 & 1.20483 & -2.79517 & -0.795166 & 0.204834 & 1.20483 & 0.204834 \\
\hline 0.204834 & -0.795166 & 0.204834 & 1.20483 & -0.795166 & 0.204834 & -0.795166 \\
\hline 0.204834 & 1.20483 & 0.204834 & 0.204834 & -1.79517 & 1.20483 & 1.20483 \\
\hline 0.204834 & -0.795166 & -1.79517 & -1.79517 & -0.795166 & -0.795166 & -0.795166 \\
\hline 0.204834 & 0.204834 & 0.204834 & 2.20483 & 0.204834 & 1.20483 & -1.79517 \\
\hline-1.79517 & -0.795166 & 1.20483 & -0.795166 & -0.795166 & -0.795166 & -0.795166 \\
\hline 1.20483 & 2.20483 & -0.795166 & -2.79517 & -0.795166 & -0.795166 & 0.204834 \\
\hline 2.20483 & 0.204834 & -0.795166 & 0.204834 & 1.20483 & 0.204834 & -0.795166 \\
\hline 0.204834 & 1.20483 & -1.79517 & 0.204834 & 1.20483 & -0.795166 & -0.795166 \\
\hline 0.204834 & 1.20483 & 0.204834 & -0.795166 & 0.204834 & 0.204834 & -0.795166 \\
\hline 0.204834 & 0.204834 & 2.20483 & 2.20483 & -0.795166 & 0.204834 & -0.795166 \\
\hline 0.204834 & -0.795166 & 0.204834 & -0.795166 & 0.204834 & -0.795166 & 0.204834 \\
\hline 1.20483 & 0.204834 & -0.795166 & 1.20483 & 1.20483 & -0.795166 & 0.204834 \\
\hline 1.20483 & -0.795166 & 0.204834 & 1.20483 & -0.795166 & 0.204834 & -0.795166 \\
\hline-0.795166 & -0.795166 & 0.204834 & -0.795166 & -0.795166 & -0.795166 & -1.79517 \\
\hline-0.795166 & -0.795166 & 0.204834 & 0.204834 & 0.204834 & -0.795166 & 0.204834 \\
\hline 0.204834 & 0.204834 & 0.204834 & -1.79517 & -0.795166 & -0.795166 & -1.79517 \\
\hline-0.795166 & -0.795166 & -0.795166 & 0.204834 & 0.204834 & -0.795166 & -1.79517 \\
\hline 1.20483 & 0.204834 & 0.204834 & 0.204834 & -0.795166 & -0.795166 & -1.79517 \\
\hline 0.204834 & 1.20483 & 1.20483 & 0.204834 & -0.795166 & -0.795166 & -0.795166 \\
\hline 2.20483 & 0.204834 & 1.20483 & 1.20483 & 1.20483 & 0.204834 & 1.20483 \\
\hline 0.204834 & 0.204834 & 0.204834 & 0.204834 & 0.204834 & 0.204834 & 0.204834 \\
\hline 0.204834 & 0.204834 & 0.204834 & 0.204834 & 1.20483 & 1.20483 & 1.20483 \\
\hline-1.79517 & 0.204834 & 1.20483 & 0.204834 & -0.795166 & -0.795166 & 1.20483 \\
\hline 0.204834 & -0.795166 & -1.79517 & 0.204834 & 2.20483 & 0.204834 & 0.204834 \\
\hline-0.795166 & -0.795166 & 0.204834 & 0.204834 & -0.795166 & 1.20483 & 0.204834 \\
\hline 0.204834 & 1.20483 & 0.204834 & -1.79517 & 0.204834 & 0.204834 & 0.204834 \\
\hline 0.204834 & -0.795166 & 1.20483 & 0.204834 & 0.204834 & 0.204834 & 0.204834 \\
\hline-1.79517 & -0.795166 & 1.20483 & 0.204834 & 0.204834 & 1.20483 & -0.795166 \\
\hline-0.795166 & 1.20483 & -0.795166 & -0.795166 & -0.795166 & 0.204834 & 1.20483 \\
\hline 1.20483 & 0.204834 & -0.795166 & -0.795166 & -0.795166 & 1.20483 & 1.20483 \\
\hline 0.204834 & 1.20483 & 0.204834 & 0.204834 & 0.204834 & -0.795166 & 0.204834 \\
\hline-0.795166 & -1.79517 & -0.795166 & -0.795166 & 1.20483 & 0.204834 & -0.795166 \\
\hline 0.204834 & -0.795166 & -1.79517 & -0.795166 & -0.795166 & 1.20483 & 0.204834 \\
\hline-0.795166 & 0.204834 & 0.204834 & -0.795166 & -0.795166 & 0.204834 & 0.204834 \\
\hline 0.204834 & -1.79517 & -0.795166 & 1.20483 & -0.795166 & 0.204834 & -0.795166 \\
\hline-1.79517 & -0.795166 & -1.79517 & -0.795166 & 1.20483 & 0.204834 & -0.795166 \\
\hline 0.204834 & -0.795166 & -0.795166 & 0.204834 & 2.20483 & -0.795166 & 1.20483 \\
\hline-0.795166 & -0.795166 & -1.79517 & -0.795166 & 0.204834 & -1.79517 & 1.20483 \\
\hline 1.20483 & 0.204834 & 0.204834 & -0.795166 & 1.20483 & 0.204834 & -0.795166 \\
\hline 0.204834 & -0.795166 & -0.795166 & -0.795166 & 0.204834 & 0.204834 & -0.795166 \\
\hline-0.795166 & -0.795166 & -0.795166 & 1.20483 & -0.795166 & 1.20483 & 0.204834 \\
\hline 0.204834 & 1.20483 & 1.20483 & -1.79517 & -0.795166 & 0.204834 & 0.204834 \\
\hline-1.79517 & -0.795166 & -0.795166 & 0.204834 & 0.204834 & -1.79517 & 0.204834 \\
\hline-0.795166 & 1.20483 & 1.20483 & -1.79517 & 0.204834 & 0.204834 & -1.79517 \\
\hline 0.204834 & -0.795166 & -0.795166 & -0.795166 & -0.795166 & -0.795166 & 0.204834 \\
\hline 0.204834 & 0.204834 & -0.795166 & -0.795166 & -0.795166 & 0.204834 & -0.795166 \\
\hline 0.204834 & -1.79517 & -0.795166 & 0.204834 & 0.204834 & 0.204834 & 0.204834 \\
\hline-0.795166 & 0.204834 & -0.795166 & 0.204834 & 0.204834 & -0.795166 & 1.20483 \\
\hline 0.204834 & 1.20483 & -0.795166 & -0.795166 & 0.204834 & 0.204834 & -0.795166 \\
\hline 0.204834 & 0.204834 & 0.204834 & -0.795166 & 1.20483 & -0.795166 & 0.204834 \\
\hline
\end{tabular}




\begin{tabular}{|c|c|c|c|c|c|c|}
\hline $\begin{array}{l}10 / 11 / 04 \\
10: 09: 30\end{array}$ & \multicolumn{5}{|c|}{ 31Steel_010_100.1st } & \\
\hline-0.795166 & -0.795166 & -0.795166 & 0.204834 & 0.204834 & -1.79517 & -0.795166 \\
\hline 1.20483 & -0.795166 & 0.204834 & 0.204834 & -0.795166 & 0.204834 & -0.795166 \\
\hline-0.795166 & -0.795166 & -1.79517 & -1.79517 & -0.795166 & -0.795166 & 2.20483 \\
\hline 0.204834 & -0.795166 & -0.795166 & -1.79517 & -0.795166 & -0.795166 & 1.20483 \\
\hline 0.204834 & -0.795166 & 0.204834 & -0.795166 & 1.20483 & -0.795166 & -2.79517 \\
\hline-1.79517 & -3.79517 & -2.79517 & -0.795166 & 0.204834 & 3.20483 & 4. 20483 \\
\hline 6.20483 & 0.204834 & -8.79517 & -9.79517 & -7.79517 & 3.20483 & 9.20483 \\
\hline 8.20483 & -1.79517 & -5.79517 & -3.79517 & 1.20483 & 1.20483 & -2.79517 \\
\hline-7.79517 & -3.79517 & 6.20483 & 13.2048 & 5.20483 & -6.79517 & -11.7952 \\
\hline-6.79517 & 4.20483 & 8.20483 & 8.20483 & -2.79517 & -5.79517 & -4.79517 \\
\hline-0.795166 & 2.20483 & 1.20483 & 2.20483 & 1.20483 & 0.204834 & 0.204834 \\
\hline-1.79517 & -1.79517 & -0.795166 & -0.795166 & 2.20483 & 1.20483 & -0.795166 \\
\hline-0.795166 & 1.20483 & 1.20483 & -1.79517 & -2.79517 & 0.204834 & 0.204834 \\
\hline 1.20483 & 1.20483 & -0.795166 & -1.79517 & -0.795166 & 1.20483 & 1.20483 \\
\hline-0.795166 & -0.795166 & 0.204834 & -0.795166 & 0.204834 & -0.795166 & -0.795166 \\
\hline 1.20483 & 2.20483 & -1.79517 & -0.795166 & 0.204834 & -0.795166 & 1.20483 \\
\hline 0.204834 & 0.204834 & -1.79517 & 1.20483 & -0.795166 & 0.204834 & 0.204834 \\
\hline-0.795166 & -1.79517 & 0.204834 & 0.204834 & -0.795166 & 0.204834 & -1.79517 \\
\hline 0.204834 & 3.20483 & 1.20483 & 0.204834 & -1.79517 & -0.795166 & -0.795166 \\
\hline 1.20483 & -0.795166 & -0.795166 & 0.204834 & 0.204834 & 1.20483 & 0.204834 \\
\hline-0.795166 & -0.795166 & -0.795166 & -0.795166 & 0.204834 & 1.20483 & 0.204834 \\
\hline 0.204834 & -0.795166 & 0.204834 & 1.20483 & 0.204834 & -0.795166 & -0.795166 \\
\hline-0.795166 & -1.79517 & -0.795166 & -1.79517 & -1.79517 & 0.204834 & 2.20483 \\
\hline 1.20483 & 1.20483 & 1.20483 & 0.204834 & -2.79517 & -2.79517 & -1.79517 \\
\hline 0.204834 & 0.204834 & 2.20483 & 2.20483 & 1.20483 & 0.204834 & -2.79517 \\
\hline-8.79517 & -9.79517 & -6.79517 & 2.20483 & 15.2048 & 30.2048 & 32.2048 \\
\hline 19.2048 & -9.79517 & -45.7952 & -69.7952 & -70.7952 & -37.7952 & 19.2048 \\
\hline 78.2048 & 111.205 & 108.205 & 55.2048 & -22.7952 & -97.7952 & -137.795 \\
\hline-124.795 & -64.7952 & 17.2048 & 92.2048 & 134.205 & 121.205 & 67.2048 \\
\hline-9.79517 & -79.7952 & -113.795 & -105.795 & -60.7952 & -1.79517 & 54.2048 \\
\hline 86.2048 & 84.2048 & 56.2048 & 11.2048 & -28.7952 & -52.7952 & -56.7952 \\
\hline-40.7952 & -14.7952 & 9.20483 & 27.2048 & 30.2048 & 23.2048 & 8.20483 \\
\hline-7.79517 & -17.7952 & -19.7952 & -12.7952 & -2.79517 & 5.20483 & 13.2048 \\
\hline 16.2048 & 10.2048 & 5.20483 & -1.79517 & -5.79517 & -10.7952 & -10.7952 \\
\hline-6.79517 & -2.79517 & 1.20483 & 3.20483 & 4.20483 & 2.20483 & -0.795166 \\
\hline-0.795166 & 0.204834 & 0.204834 & 0.204834 & 1.20483 & 1.20483 & 1.20483 \\
\hline 0.204834 & -1.79517 & -0.795166 & -2.79517 & -1.79517 & -3.79517 & -2.79517 \\
\hline-2.79517 & -0.795166 & 0.204834 & 1.20483 & 1.20483 & 1.20483 & 1.20483 \\
\hline 3.20483 & 0.204834 & -1.79517 & 0.204834 & -0.795166 & 0.204834 & -0.795166 \\
\hline-2.79517 & -0.795166 & -0.795166 & 1.20483 & 0.204834 & 1.20483 & 1.20483 \\
\hline-0.795166 & 0.204834 & 2.20483 & 0.204834 & 1.20483 & 2.20483 & 0.204834 \\
\hline-0.795166 & -0.795166 & 0.204834 & -0.795166 & -0.795166 & -0.795166 & 0.204834 \\
\hline 1.20483 & 1.20483 & 1.20483 & 1.20483 & 0.204834 & -1.79517 & 1.20483 \\
\hline 3.20483 & -0.795166 & -1.79517 & 1.20483 & 0.204834 & 2.20483 & 1.20483 \\
\hline-0.795166 & -2.79517 & -1.79517 & -0.795166 & 0.204834 & -0.795166 & 0.204834 \\
\hline 0.204834 & 0.204834 & 1.20483 & 1.20483 & 1.20483 & 1.20483 & -1.79517 \\
\hline-1.79517 & -0.795166 & -0.795166 & -1.79517 & -0.795166 & -2.79517 & 0.204834 \\
\hline 2.20483 & 3.20483 & 3.20483 & -0.795166 & -3.79517 & -3.79517 & -1.79517 \\
\hline 0.204834 & 2.20483 & 0.204834 & -0.795166 & -0.795166 & -0.795166 & 2.20483 \\
\hline 0.204834 & 0.204834 & -0.795166 & 0.204834 & 0.204834 & 0.204834 & -0.795166 \\
\hline-0.795166 & -0.795166 & 0.204834 & 0.204834 & -0.795166 & -1.79517 & -0.795166 \\
\hline-0.795166 & 0.204834 & 0.204834 & -0.795166 & 1.20483 & 0.204834 & 0.204834 \\
\hline-0.795166 & 0.204834 & -0.795166 & 0.204834 & -0.795166 & 0.204834 & 1.20483 \\
\hline 0.204834 & 0.204834 & 1.20483 & 1.20483 & -0.795166 & 1.20483 & -0.795166 \\
\hline 0.204834 & 0.204834 & -2.79517 & 0.204834 & 1.20483 & -0.795166 & -0.795166 \\
\hline-1.79517 & 1.20483 & 0.204834 & 1.20483 & 1.20483 & -1.79517 & -0.795166 \\
\hline 0.204834 & 3.20483 & 2.20483 & -0.795166 & -1.79517 & -1.79517 & 0.204834 \\
\hline 0.204834 & -0.795166 & 0.204834 & 1.20483 & 1.20483 & 1.20483 & -0.795166 \\
\hline-1.79517 & 0.204834 & 2.20483 & 1.20483 & -2.79517 & -2.79517 & -2.79517 \\
\hline 1.20483 & 2.20483 & 2.20483 & -1.79517 & -3.79517 & -0.795166 & -0.795166 \\
\hline 1.20483 & 0.204834 & 1.20483 & 0.204834 & 1.20483 & -1.79517 & -1.79517 \\
\hline-0.795166 & -2.79517 & 1.20483 & 1.20483 & -0.795166 & -0.795166 & -0.795166 \\
\hline-2.79517 & -0.795166 & 0.204834 & -0.795166 & -0.795166 & -0.795166 & 0.204834 \\
\hline 2.20483 & -0.795166 & 0.204834 & -0.795166 & 1.20483 & 0.204834 & 0.204834 \\
\hline-1.79517 & 0.204834 & -0.795166 & 0.204834 & -1.79517 & -0.795166 & -0.795166 \\
\hline 1.20483 & 3.20483 & -0.795166 & -5.79517 & -3.79517 & 3.20483 & 6.20483 \\
\hline 6.20483 & -1.79517 & -4.79517 & -2.79517 & -0.795166 & 0.204834 & 3.20483 \\
\hline 3.20483 & 2.20483 & -1.79517 & -3.79517 & -2.79517 & -0.795166 & 2.20483 \\
\hline
\end{tabular}




\begin{tabular}{|c|c|c|c|c|c|c|}
\hline \multirow{2}{*}{$\begin{array}{r}\text { 10/11/04 } \\
10: 09: 30 \\
4.20483\end{array}$} & \multicolumn{5}{|c|}{ 31Steel_010_100.1st } & \\
\hline & 2.20483 & -3.79517 & -7.79517 & -1.79517 & 3.20483 & 8.20483 \\
\hline 7.20483 & 0.204834 & -3.79517 & -4.79517 & -3.79517 & -2.79517 & 0.204834 \\
\hline 0.204834 & 0.204834 & 0.204834 & -0.795166 & -0.795166 & -0.795166 & 2.20483 \\
\hline 0.204834 & -1.79517 & -8.79517 & -5.79517 & 4. 20483 & 8. 20483 & 8.20483 \\
\hline-2.79517 & -3.79517 & -5.79517 & -2.79517 & -1.79517 & 0.204834 & 5.20483 \\
\hline 4. 20483 & 2.20483 & -0.795166 & -3.79517 & -1.79517 & 1.20483 & 6.20483 \\
\hline 2.20483 & -2.79517 & -5.79517 & -4.79517 & 2.20483 & 6.20483 & 5.20483 \\
\hline 0.204834 & -3.79517 & -4.79517 & -0.795166 & -0.795166 & -0.795166 & 0.204834 \\
\hline 1.20483 & 1.20483 & 1.20483 & -0.795166 & 0.204834 & -2.79517 & -0.795166 \\
\hline-1.79517 & 0.204834 & 0.204834 & 2.20483 & 2.20483 & 0.204834 & -2.79517 \\
\hline-0.795166 & -0.795166 & 1.20483 & 1.20483 & 1.20483 & 0.204834 & -1.79517 \\
\hline-1.79517 & 0.204834 & 2.20483 & 4.20483 & 2.20483 & -3.79517 & -6.79517 \\
\hline-2.79517 & 1.20483 & 4. 20483 & 9.20483 & 10.2048 & 4. 20483 & -4.79517 \\
\hline-15.7952 & -22.7952 & -12.7952 & 11.2048 & 32.2048 & 30.2048 & 8. 20483 \\
\hline-22.7952 & -32.7952 & -22.7952 & -0.795166 & 20.2048 & 30.2048 & 17.2048 \\
\hline-4.79517 & -22.7952 & -22.7952 & -6.79517 & 13.2048 & 23.2048 & 13.2048 \\
\hline-1.79517 & -17.7952 & -18.7952 & -3.79517 & 15.2048 & 22.2048 & 10.2048 \\
\hline-19.7952 & -34.7952 & -16.7952 & 22.2048 & 44.2048 & 33.2048 & 0.204834 \\
\hline-28.7952 & -46.7952 & -37.7952 & -3.79517 & 38.2048 & 57.2048 & 40.2048 \\
\hline 0.204834 & -37.7952 & -48.7952 & -30.7952 & 11.2048 & 41.2048 & 39.2048 \\
\hline 12.2048 & -14.7952 & -27.7952 & -25.7952 & -12.7952 & 2.20483 & 20.2048 \\
\hline 33.2048 & 28.2048 & 6.20483 & -15.7952 & -28.7952 & -27.7952 & -16.7952 \\
\hline 0.204834 & 17.2048 & 33.2048 & 35.2048 & 24.2048 & -7.79517 & -43.7952 \\
\hline-55.7952 & -28.7952 & 18.2048 & 49.2048 & 49.2048 & 21.2048 & -17.7952 \\
\hline-44.7952 & -49.7952 & -30.7952 & 11.2048 & 46.2048 & 60.2048 & 39.2048 \\
\hline-6.79517 & -52.7952 & -68.7952 & -45.7952 & 3.20483 & 49.2048 & 61.2048 \\
\hline 37.2048 & -0.795166 & -30.7952 & -41.7952 & -29.7952 & -8.79517 & 12.2048 \\
\hline 20.2048 & 22.2048 & 12.2048 & 2.20483 & -6.79517 & -11.7952 & -7.79517 \\
\hline-5.79517 & -6.79517 & -0.795166 & 2.20483 & 8.20483 & 14.2048 & 8. 20483 \\
\hline-0.795166 & -5.79517 & -6.79517 & -5.79517 & -6.79517 & -8.79517 & -1.79517 \\
\hline 12.2048 & 27.2048 & 20.2048 & 0.204834 & -20.7952 & -30.7952 & -22.7952 \\
\hline-3.79517 & 13.2048 & 26.2048 & 25.2048 & 15.2048 & -5.79517 & -26.7952 \\
\hline-29.7952 & -13.7952 & 10.2048 & 22.2048 & 19.2048 & 7.20483 & -4.79517 \\
\hline-8.79517 & -1.79517 & -0.795166 & -6.79517 & -11.7952 & -6.79517 & 8. 20483 \\
\hline 18.2048 & 15.2048 & 2.20483 & -7.79517 & -10.7952 & -12.7952 & -9.79517 \\
\hline-3.79517 & 3.20483 & 11.2048 & 14.2048 & 9.20483 & 1.20483 & -2.79517 \\
\hline-5.79517 & -4.79517 & -5.79517 & -11.7952 & -13.7952 & -3.79517 & 12.2048 \\
\hline 23.2048 & 20.2048 & 4. 20483 & -14.7952 & -24.7952 & -16.7952 & -2.79517 \\
\hline 10.2048 & 11.2048 & 1.20483 & -2.79517 & -3.79517 & 1.20483 & 10.2048 \\
\hline 10.2048 & -2.79517 & -8.79517 & -15.7952 & -11.7952 & -4.79517 & 10.2048 \\
\hline 19.2048 & 17.2048 & 8.20483 & -6.79517 & -16.7952 & -13.7952 & -5.79517 \\
\hline 8.20483 & 9.20483 & 8.20483 & 4. 20483 & 0.204834 & 1.20483 & 2.20483 \\
\hline 1.20483 & -1.79517 & -6.79517 & -5.79517 & -1.79517 & 5.20483 & 13.2048 \\
\hline 12.2048 & 6.20483 & -3.79517 & -9.79517 & -9.79517 & -3.79517 & 1.20483 \\
\hline 7.20483 & 11.2048 & 7. 20483 & 4. 20483 & 0.204834 & -2.79517 & -2.79517 \\
\hline-0.795166 & -2.79517 & -0.795166 & 2.20483 & 4.20483 & 4. 20483 & 3.20483 \\
\hline-0.795166 & -2.79517 & -3.79517 & -2.79517 & 0.204834 & 2.20483 & 3.20483 \\
\hline 3.20483 & 0.204834 & -0.795166 & -2.79517 & 0.204834 & 2.20483 & 3.20483 \\
\hline 1.20483 & 1.20483 & -1.79517 & -1.79517 & -3.79517 & -3.79517 & -1.79517 \\
\hline 0.204834 & 7.20483 & 8.20483 & 4.20483 & -2.79517 & -8.79517 & -11.7952 \\
\hline-6.79517 & 0.204834 & 8.20483 & 8.20483 & 6.20483 & 1.20483 & -7.79517 \\
\hline-9.79517 & -8.79517 & -1.79517 & 5.20483 & 7.20483 & 7.20483 & 0.204834 \\
\hline-3.79517 & -8.79517 & -1.79517 & 3.20483 & 5.20483 & 7.20483 & -0.795166 \\
\hline-4.79517 & -4.79517 & -2.79517 & 1.20483 & 5.20483 & 8.20483 & 4. 20483 \\
\hline-3.79517 & -2.79517 & -3.79517 & -0.795166 & 3.20483 & 5.20483 & 2.20483 \\
\hline-1.79517 & -2.79517 & -1.79517 & 3. 20483 & 3.20483 & 2.20483 & -0.795166 \\
\hline-1.79517 & -3.79517 & -3.79517 & -1.79517 & 0.204834 & 4. 20483 & 5.20483 \\
\hline 2. 20483 & 1.20483 & 3. 20483 & 4. 20483 & -0.795166 & -7.79517 & -10.7952 \\
\hline-6.79517 & 6.20483 & 10.2048 & 7.20483 & 1. 20483 & -0.795166 & 3.20483 \\
\hline 1.20483 & -2.79517 & -9.79517 & -10.7952 & 0.204834 & 11.2048 & 14.2048 \\
\hline 5.20483 & -3.79517 & -5.79517 & 0.204834 & 1.20483 & -0.795166 & -8.79517 \\
\hline-6.79517 & -1.79517 & 6.20483 & 9.20483 & 5.20483 & -0.795166 & -2.79517 \\
\hline-2.79517 & -2.79517 & -4.79517 & -3.79517 & -3.79517 & 1. 20483 & 6.20483 \\
\hline 2.20483 & 1.20483 & 0.204834 & 1.20483 & -1.79517 & -2.79517 & -6.79517 \\
\hline-4.79517 & 2.20483 & 6.20483 & 6.20483 & 3.20483 & -1.79517 & -3.79517 \\
\hline-5.79517 & -1.79517 & 1.20483 & 2.20483 & 1.20483 & 3.20483 & -0.795166 \\
\hline 0.204834 & -1.79517 & -1.79517 & 0.204834 & 3.20483 & 2.20483 & 0.204834 \\
\hline-0.795166 & 0.204834 & -0.795166 & -1.79517 & -1.79517 & -2.79517 & -0.795166 \\
\hline
\end{tabular}




\begin{tabular}{|c|c|c|c|c|c|c|}
\hline $\begin{array}{l}10 / 11 / 04 \\
10: 09: 30\end{array}$ & & & Steel_010_ & 00.1 st & & \\
\hline 1.20483 & 3.20483 & 4.20483 & 3.20483 & 2.20483 & -1.79517 & -3.79517 \\
\hline-5.79517 & -3.79517 & 0.204834 & 4.20483 & 5.20483 & 6.20483 & 2.20483 \\
\hline-2.79517 & -4.79517 & -3.79517 & -1.79517 & 1.20483 & 4.20483 & 3.20483 \\
\hline 2.20483 & -1.79517 & -0.795166 & 0.204834 & 2.20483 & 2.20483 & 3.20483 \\
\hline-0.795166 & -6.79517 & -6.79517 & -1.79517 & 0.204834 & 6.20483 & 7.20483 \\
\hline 5. 20483 & 1.20483 & -4.79517 & -7.79517 & -7.79517 & -3.79517 & 1.20483 \\
\hline 6.20483 & 6.20483 & 3.20483 & 1.20483 & -1.79517 & -4.79517 & -6.79517 \\
\hline-4.79517 & -1.79517 & 3.20483 & 4.20483 & 5.20483 & 6.20483 & -0.795166 \\
\hline-2.79517 & -5.79517 & -5.79517 & -3.79517 & 1.20483 & 3.20483 & 6.20483 \\
\hline 5.20483 & 3.20483 & -2.79517 & -3.79517 & -3.79517 & -2.79517 & -0.795166 \\
\hline 2.20483 & 4.20483 & 4.20483 & 6.20483 & 1.20483 & -2.79517 & -4.79517 \\
\hline-2.79517 & -0.795166 & 3.20483 & 3.20483 & 2.20483 & 0.204834 & -2.79517 \\
\hline-0.795166 & -0.795166 & 1.20483 & 3.20483 & 3.20483 & 2.20483 & -1.79517 \\
\hline-2.79517 & -2.79517 & 0.204834 & 2.20483 & 3.20483 & 2.20483 & 1. 20483 \\
\hline-1.79517 & -1.79517 & 2.20483 & -0.795166 & -0.795166 & 1.20483 & 2.20483 \\
\hline 3.20483 & 1.20483 & 0.204834 & -1.79517 & -0.795166 & 0.204834 & 0.204834 \\
\hline 0.204834 & 0.204834 & 0.204834 & 0.204834 & 0.204834 & -0.795166 & 0.204834 \\
\hline 2.20483 & 1.20483 & 1.20483 & -0.795166 & -3.79517 & -0.795166 & 0.204834 \\
\hline 1.20483 & 1.20483 & 1.20483 & 0.204834 & 0.204834 & 1.20483 & 3.20483 \\
\hline 2.20483 & -2.79517 & -4.79517 & -4.79517 & 2.20483 & 5.20483 & 7.20483 \\
\hline 3.20483 & -0.795166 & -4.79517 & -5.79517 & -3.79517 & 0.204834 & 2.20483 \\
\hline 5.20483 & 6.20483 & 3.20483 & -3.79517 & -5.79517 & -2.79517 & 0.204834 \\
\hline 3.20483 & 2.20483 & 2.20483 & 1.20483 & 0.204834 & -0.795166 & 0.204834 \\
\hline 1.20483 & -0.795166 & -0.795166 & -0.795166 & 0.204834 & 2. 20483 & 1.20483 \\
\hline 0.204834 & -0.795166 & 0.204834 & -0.795166 & -1.79517 & -0.795166 & 1.20483 \\
\hline 0.204834 & 2.20483 & -1.79517 & 0.204834 & -1.79517 & 0.204834 & -0.795166 \\
\hline-1.79517 & -0.795166 & -0.795166 & 1.20483 & 0.204834 & 2.20483 & -0.795166 \\
\hline-0.795166 & 0.204834 & -2.79517 & -0.795166 & 1. 20483 & 1.20483 & 3.20483 \\
\hline 2. 20483 & -1.79517 & -1.79517 & -0.795166 & -3.79517 & -0.795166 & 1.20483 \\
\hline 1.20483 & 2.20483 & 0.204834 & -1.79517 & -0.795166 & -2.79517 & -0.795166 \\
\hline-2.79517 & -0.795166 & -0.795166 & 1.20483 & 1. 20483 & 1.20483 & -0.795166 \\
\hline-2.79517 & -2.79517 & 0.204834 & 0.204834 & 1.20483 & 1.20483 & 1. 20483 \\
\hline 1.20483 & -0.795166 & -2.79517 & -2.79517 & -1.79517 & 2.20483 & 3.20483 \\
\hline 4.20483 & 3.20483 & 0.204834 & -1.79517 & -0.795166 & 0.204834 & 0.204834 \\
\hline 2.20483 & 2.20483 & 2.20483 & 1.20483 & 0.204834 & 1.20483 & 0.204834 \\
\hline 1.20483 & 0.204834 & 1.20483 & -0.795166 & 0.204834 & 0.204834 & -1.79517 \\
\hline-0.795166 & -0.795166 & 0.204834 & 2. 20483 & 1.20483 & 1.20483 & 0.204834 \\
\hline 0.204834 & -0.795166 & 0.204834 & 2.20483 & 3.20483 & 2.20483 & -0.795166 \\
\hline 4.20483 & -0.795166 & -0.795166 & 0.204834 & -0.795166 & 0.204834 & 0.204834 \\
\hline 0.204834 & 0.204834 & -0.795166 & 2.20483 & 2.20483 & 1.20483 & 0.204834 \\
\hline-2.79517 & -4.79517 & 2.20483 & 2.20483 & 0.204834 & 1.20483 & 1.20483 \\
\hline-0.795166 & -2.79517 & -2.79517 & -1.79517 & 0.204834 & 2.20483 & 3.20483 \\
\hline 1.20483 & -2.79517 & -1.79517 & 1.20483 & 0.204834 & 3.20483 & -0.795166 \\
\hline-0.795166 & -1.79517 & 1.20483 & 2.20483 & -0.795166 & -0.795166 & -0.795166 \\
\hline 0.204834 & 2.20483 & 1.20483 & 0.204834 & 0.204834 & 0.204834 & 1. 20483 \\
\hline 0.204834 & -0.795166 & -0.795166 & 0.204834 & -0.795166 & 0.204834 & 1.20483 \\
\hline 1.20483 & 1.20483 & 2.20483 & -0.795166 & -2.79517 & -0.795166 & -0.795166 \\
\hline 0.204834 & -0.795166 & 0.204834 & 0.204834 & -0.795166 & -0.795166 & 0.204834 \\
\hline 0.204834 & -0.795166 & 0.204834 & 2.20483 & -0.795166 & 2. 20483 & 1.20483 \\
\hline-1.79517 & -0.795166 & 0.204834 & -0.795166 & 2.20483 & 3.20483 & -0.795166 \\
\hline-0.795166 & -1.79517 & -2.79517 & -1.79517 & -0.795166 & 2.20483 & 2.20483 \\
\hline 3. 20483 & -0.795166 & -1.79517 & -2.79517 & -2.79517 & -0.795166 & 0.204834 \\
\hline 1.20483 & 0.204834 & 0.204834 & -0.795166 & 0.204834 & -1.79517 & -1.79517 \\
\hline-1.79517 & 1.20483 & 1.20483 & -0.795166 & -0.795166 & -2.79517 & -2.79517 \\
\hline 1.20483 & 1.20483 & 1.20483 & -0.795166 & -1.79517 & 0.204834 & -0.795166 \\
\hline 0.204834 & 0.204834 & -0.795166 & 0.204834 & 0.204834 & 0.204834 & 1.20483 \\
\hline 1.20483 & -0.795166 & 0.204834 & -0.795166 & 0.204834 & 0.204834 & 0.204834 \\
\hline 0.204834 & 0.204834 & 0.204834 & -0.795166 & 0.204834 & -1.79517 & 0.204834 \\
\hline 0.204834 & 1.20483 & 0.204834 & 0.204834 & -2.79517 & 0.204834 & 1.20483 \\
\hline 0.204834 & -0.795166 & 0.204834 & 1.20483 & 1.20483 & 0.204834 & -0.795166 \\
\hline-0.795166 & 0.204834 & 1.20483 & 0.204834 & -0.795166 & -1.79517 & 0.204834 \\
\hline 1.20483 & 0.204834 & -0.795166 & 0.204834 & 2. 20483 & 2.20483 & 0.204834 \\
\hline-0.795166 & -0.795166 & -1.79517 & -0.795166 & 1.20483 & 2.20483 & 1.20483 \\
\hline 1. 20483 & 0.204834 & -0.795166 & -0.795166 & -0.795166 & -0.795166 & -0.795166 \\
\hline 0.204834 & 1.20483 & 1.20483 & 0.204834 & -0.795166 & -0.795166 & -0.795166 \\
\hline 0.204834 & -0.795166 & -1.79517 & -0.795166 & 0.204834 & -0.795166 & 0.204834 \\
\hline-1.79517 & 0.204834 & 0.204834 & 0.204834 & -0.795166 & 0.204834 & -0.795166 \\
\hline-0.795166 & 1.20483 & 0.204834 & -0.795166 & 1. 20483 & -0.795166 & -0.795166 \\
\hline
\end{tabular}




\begin{tabular}{|c|c|c|c|c|c|c|}
\hline $\begin{array}{l}10 / 11 / 04 \\
10: 09: 30\end{array}$ & & & Steel_010_ & 00.1 st & & \\
\hline 0.204834 & 1.20483 & -0.795166 & -0.795166 & -0.795166 & 2.20483 & -1.79517 \\
\hline 1.20483 & 0.204834 & 0.204834 & 0.204834 & 0.204834 & -0.795166 & -0.795166 \\
\hline-0.795166 & 1.20483 & 0.204834 & 0.204834 & -1.79517 & 0.204834 & 0.204834 \\
\hline-0.795166 & -0.795166 & -0.795166 & 0.204834 & 0.204834 & 1.20483 & 0.204834 \\
\hline 0.204834 & 1.20483 & -1.79517 & 1.20483 & 0.204834 & -1.79517 & -0.795166 \\
\hline-0.795166 & 0.204834 & -0.795166 & 0.204834 & -0.795166 & -0.795166 & 0.204834 \\
\hline 0.204834 & 2.20483 & 0.204834 & -0.795166 & 0.204834 & -0.795166 & 1.20483 \\
\hline 0.204834 & 0.204834 & -0.795166 & -0.795166 & -0.795166 & 0.204834 & 0.204834 \\
\hline 0.204834 & 0.204834 & 0.204834 & 1.20483 & 0.204834 & -0.795166 & 0.204834 \\
\hline-0.795166 & 1.20483 & -0.795166 & -0.795166 & 0.204834 & 0.204834 & 2.20483 \\
\hline 1.20483 & 0.204834 & -0.795166 & -0.795166 & 1.20483 & 0.204834 & 2.20483 \\
\hline 1.20483 & 0.204834 & -0.795166 & 0.204834 & 0.204834 & -0.795166 & 0.204834 \\
\hline 1.20483 & 0.204834 & -1.79517 & 0.204834 & -0.795166 & 0.204834 & 0.204834 \\
\hline 1.20483 & -0.795166 & 0.204834 & -1.79517 & 0.204834 & 1.20483 & -1.79517 \\
\hline 0.204834 & 0.204834 & -0.795166 & -0.795166 & 0.204834 & 1.20483 & -1.79517 \\
\hline-0.795166 & -0.795166 & 1.20483 & 1.20483 & 3.20483 & 0.204834 & -1.79517 \\
\hline 1.20483 & 0.204834 & -0.795166 & -1.79517 & 1.20483 & 0.204834 & 0.204834 \\
\hline 0.204834 & 0.204834 & -1.79517 & -0.795166 & 1.20483 & 0.204834 & 1.20483 \\
\hline-1.79517 & -0.795166 & 1.20483 & 2.20483 & 1.20483 & -0.795166 & 0.204834 \\
\hline 0.204834 & 0.204834 & -0.795166 & 1.20483 & 0.204834 & 0.204834 & 3.20483 \\
\hline 0.204834 & 0.204834 & -1.79517 & -0.795166 & 0.204834 & 0.204834 & -0.795166 \\
\hline 1.20483 & 0.204834 & 1.20483 & 0.204834 & -0.795166 & -0.795166 & 0.204834 \\
\hline 0.204834 & 2.20483 & -0.795166 & 0.204834 & -0.795166 & -0.795166 & 1.20483 \\
\hline 1.20483 & 0.204834 & -0.795166 & -0.795166 & 0.204834 & 2.20483 & 1.20483 \\
\hline-0.795166 & 0.204834 & 1.20483 & 1.20483 & 0.204834 & -0.795166 & -1.79517 \\
\hline 0.204834 & -0.795166 & -0.795166 & -0.795166 & -0.795166 & -0.795166 & 0.204834 \\
\hline-0.795166 & 0.204834 & -0.795166 & 0.204834 & -0.795166 & -0.795166 & -0.795166 \\
\hline 0.204834 & -0.795166 & -0.795166 & -1.79517 & -0.795166 & -0.795166 & -1.79517 \\
\hline 0.204834 & 0.204834 & 0.204834 & -0.795166 & 0.204834 & 0.204834 & 0.204834 \\
\hline 0.204834 & -0.795166 & -0.795166 & -1.79517 & -0.795166 & -0.795166 & 0.204834 \\
\hline 0.204834 & 1.20483 & -0.795166 & 2.20483 & 0.204834 & -1.79517 & -0.795166 \\
\hline-1.79517 & 0.204834 & 1.20483 & 0.204834 & 1.20483 & -1.79517 & -0.795166 \\
\hline 0.204834 & 1.20483 & 0.204834 & -0.795166 & 0.204834 & 1.20483 & 0.204834 \\
\hline 0.204834 & 1.20483 & -0.795166 & 0.204834 & 0.204834 & 0.204834 & 2.20483 \\
\hline 1.20483 & -0.795166 & 0.204834 & 0.204834 & 2.20483 & -0.795166 & -0.795166 \\
\hline 2.20483 & 1.20483 & 2.20483 & -0.795166 & -0.795166 & 0.204834 & 0.204834 \\
\hline 1.20483 & 0.204834 & 0.204834 & 1.20483 & 0.204834 & -0.795166 & 1.20483 \\
\hline 0.204834 & 1.20483 & -0.795166 & -0.795166 & -0.795166 & -0.795166 & 1.20483 \\
\hline-0.795166 & 1.20483 & 0.204834 & 2.20483 & -0.795166 & -0.795166 & 2.20483 \\
\hline 0.204834 & 0.204834 & -0.795166 & -0.795166 & -0.795166 & -1.79517 & -2.79517 \\
\hline $\begin{array}{l}-1.79517 \\
-5.79517\end{array}$ & 2.20483 & 3.20483 & 5.20483 & 2.20483 & -2.79517 & -5.79517 \\
\hline
\end{tabular}

